\title{
Paper
}

\section{Random attractors for stochastic lattice systems with non-Lipschitz non-linearity}

\author{
T. Caraballo ${ }^{\mathrm{a} *}$, F. Morillas ${ }^{\mathrm{b}}$ and J. Valero ${ }^{\mathrm{c}}$ \\ ${ }^{a}$ Dpto. Ecuaciones Diferenciales y Análisis Numérico, Universidad de Sevilla, Apdo. de \\ Correos 1160, 41080-Sevilla, Spain.; \\ ${ }^{\mathrm{b}}$ Universitat de València, Departament d'Economia Aplicada, Facultat d'Economia, \\ Campus dels Tarongers s/n, 46022 València, Spain. \\ ${ }^{\mathrm{c}}$ Universidad Miguel Hernández, Centro de Investigación Operativa, Avda. Universidad \\ s/n, 03202 Elche (Alicante), Spain.
}

$(X X X X X X)$

\begin{abstract}
In this paper we study the asymptotic behaviour of solutions of a first-order stochastic lattice dynamical system with an additive noise.

We do not assume any Lipschitz condition on the nonlinear term, just a continuity assumption together with growth and dissipative conditions, so that uniqueness of the Cauchy problem fails to be true.

Using the theory of multi-valued random dynamical systems we prove the existence of a random compact global attractor.
\end{abstract}

Keywords: Stochastic lattice dynamical systems, set-valued dynamical system, random attractor

AMS Subject Classification: 35B40, 35B41, 35K40, 35K57, 37B25, 37L55, 60H10

\section{Introduction}

In this paper we study the asymptotic behaviour of solutions of the stochastic first order lattice dynamical system with an additive noise given by

$$
\left\{\begin{array}{l}
\frac{d u_{i}}{d t}=\nu\left(u_{i-1}-2 u_{i}+u_{i+1}\right)-h_{i}\left(u_{i}\right)-f_{i}\left(u_{i}\right)+a_{i} \frac{d w_{i}(t)}{d t}, i \in \mathbb{Z} \\
u_{i}(0)=u_{i}^{0}
\end{array}\right.
$$

under some growth and dissipative conditions on the nonlinear terms $f$ and $h$. Here $w_{i}(t)$ are real independent two-sided Brownian motions.

We prove the existence of a random global pullback attractor for such systems, extending in this way the results given in [3] (see [11] for the case of a multiplicative noise, and also [21] for a partial dissipative stochastic lattice dynamical system with additive noise). The main difference with [3] is the fact that we do not assume conditions ensuring the uniqueness of solutions for the Cauchy problem. Hence, we use the theory of multi-valued random dynamical systems [6] in order to prove the existence of the pullback attractor. Comparing our results with the single-valued

*Corresponding author. Email: caraball@us.es 
case from [3], the main technical difficulty appears in proving the measurability of the attractor.

Our stochastic lattice dynamical system can be obtained by the spatial discretization of a stochastic reaction-diffusion equation in an arbitrary domain for the spatial variable (which can be unbounded and even the whole space $\mathbb{R}^{N}$ ). So, such systems can be viewed as approximations for reaction-diffusion equations in unbounded domains.

In the deterministic case the theory of attractors for lattice dynamical systems has been intensively studied over the last years (see e.g. [1], [4], [5], [14], [15], [17], [18], [20], [22], [23], [24] and the references therein).

This paper is organized as follows.

In Section 2 we recall the general theory of multi-valued random dynamical systems as developed in [6]. First, we establish some preliminaries concerning the definitions of multi-valued non-autonomous dynamical systems (MNDS) and multivalued random dynamical systems (MRDS) which turns to be an MNDS with an additional measurability property. Then, we recall two theorems ensuring the existence of the global pullback attractor for an MNDS and the random global pullback attractor for an MRDS.

In Section 3 we describe the setting of our problem. By a standard change of variable (involving the Ornstein-Uhlenbeck process), we transform the stochastic equation into a random one depending on a parameter $\omega$. We then show that the Cauchy problem for the transformed lattice system possesses, at least, one global solution for each $\omega$, although it can be non-unique.

In Section 4 we first define an MNDS associated to our problem, and prove the existence of a global pullback attractor. In this part it is crucial to obtain suitable estimates of the tails of solutions leading to the pullback asymptotic compactness of the MNDS. Finally, we prove some measurability properties of the MNDS which imply that we have in fact a MRDS having a random pullback attractor. As remarked before, these last results are much more difficult to obtain than in the single-valued case.

\section{Multi-valued random dynamical systems}

We recall now some basic definitions for set-valued non-autonomous and random dynamical systems and formulate sufficient conditions ensuring the existence of a pullback attractor for these systems.

A pair $(\Omega, \theta)$ where $\theta=\left(\theta_{t}\right)_{t \in \mathbb{R}}$ is a flow on $\Omega$, that is,

$$
\begin{aligned}
& \theta: \mathbb{R} \times \Omega \rightarrow \Omega, \\
& \theta_{0}=\operatorname{id}_{\Omega}, \quad \theta_{t+\tau}=\theta_{t} \circ \theta_{\tau}=: \theta_{t} \theta_{\tau} \quad \text { for } t, \tau \in \mathbb{R},
\end{aligned}
$$

is called a non-autonomous perturbation.

Let $\mathcal{P}:=(\Omega, \mathcal{F}, \mathbb{P})$ be a probability space. On this probability space we consider a measurable non-autonomous flow $\theta$ :

$$
\theta:(\mathbb{R} \times \Omega, \mathcal{B}(\mathbb{R}) \otimes \mathcal{F}) \rightarrow(\Omega, \mathcal{F})
$$

In addition, $\mathbb{P}$ is supposed to be ergodic with respect to $\theta$, which means that every $\theta_{t}$-invariant set has measure zero or one for $t \in \mathbb{R}$. Hence $\mathbb{P}$ is invariant with respect to $\theta_{t}$. The quadruple $(\Omega, \mathcal{F}, \mathbb{P}, \theta)$, which is the model for a noise, is called a metric dynamical system. 
If we replace in the definition of a metric dynamical system the probability space $\mathcal{P}$ by its completion $\mathcal{P}^{c}:=(\Omega, \overline{\mathcal{F}}, \overline{\mathbb{P}})$ the above measurability property is not true in general (see Arnold [2, Appendix A]). But for fixed $t \in \mathbb{R}$ we have that the mapping

$$
\theta_{t}:(\Omega, \overline{\mathcal{F}}) \rightarrow(\Omega, \overline{\mathcal{F}})
$$

is measurable.

Let $X=\left(X, d_{X}\right)$ be a Polish space. Let $D: \omega \rightarrow D(\omega) \in 2^{X}$ be a multivalued mapping. The set of multi-functions $D: \omega \rightarrow D(\omega) \in 2^{X}$ with closed and non-empty images is denoted by $C(X)$. Let also denote by $P_{f}(X)$ the set of all non-empty closed subsets of the space $X$. Thus, it is equivalent to write that $D$ is in $C(X)$, or $D: \Omega \rightarrow P_{f}(X)$.

Let $D: \omega \rightarrow D(\omega)$ be a multi-valued mapping in $X$ over $\mathcal{P}$. Such a mapping is called a random set if

$$
\omega \rightarrow \inf _{y \in D(\omega)} d_{X}(x, y)
$$

is a random variable for every $x \in X$. It is well known that a mapping is a random set if and only if for every open set $O$ in $X$ the inverse image $\{\omega: D(\omega) \cap O \neq \emptyset\}$ is measurable, i.e., it belongs to $\mathcal{F}$ [13, Proposition 2.1.4].

Clearly, this is also valid if we replace $\mathcal{P}$ by $\mathcal{P}^{c}$ and $\mathcal{F}$ by $\overline{\mathcal{F}}$. It is straightforward to see that, if $D$ is a random set with respect to $\mathcal{P}$, then it is also random with respect to $\mathcal{P}^{c}$.

We now introduce non-autonomous and random dynamical systems.

Definition 2.1. A multi-valued map $G: \mathbb{R}^{+} \times \Omega \times X \rightarrow P_{f}(X)$ is called a multi-valued non-autonomous dynamical system (MNDS) if

i) $G(0, \omega, \cdot)=\operatorname{id}_{X}$,

ii) $G(t+\tau, \omega, x) \subset G\left(t, \theta_{\tau} \omega, G(\tau, \omega, x)\right) \quad$ (cocycle property) for all $t, \tau \in \mathbb{R}^{+}, x \in$ $X, \omega \in \Omega$.

It is called a strict MNDS if, moreover,

iii) $G(t+\tau, \omega, x)=G\left(t, \theta_{\tau} \omega, G(\tau, \omega, x)\right)$ for all $t, \tau \in \mathbb{R}^{+}, x \in X, \omega \in \Omega$.

An MNDS is called a multi-valued random dynamical system (MRDS) if the multi-valued mapping

$$
(t, \omega, x) \rightarrow G(t, \omega, x)
$$

is $\mathcal{B}\left(\mathbb{R}^{+}\right) \otimes \mathcal{F} \otimes \mathcal{B}(X)$ measurable, i.e. $\{(t, \omega, x): G(t, \omega, x) \cap O \neq \emptyset\} \in \mathcal{B}\left(\mathbb{R}^{+}\right) \otimes$ $\mathcal{F} \otimes \mathcal{B}(X)$ for every open set $O$ of the topological space $X$.

For the above composition of multi-valued mappings we use that for any nonempty set $V \subset X, G(t, \omega, V)$ is defined by

$$
G(t, \omega, V)=\bigcup_{x_{0} \in V} G\left(t, \omega, x_{0}\right)
$$

We now formulate a general condition ensuring that an MNDS defines an MRDS. We need the particular assumption that $\Omega$ is a Polish space and $\mathcal{F}$ the associated Borel $\sigma$-algebra. 
Lemma 2.2. [6, Lemma 2.5] Let $\Omega$ be a Polish space and let $\mathcal{F}$ be the Borel $\sigma$-algebra. Suppose that $(t, \omega, x) \mapsto U(t, \omega, x)$ is upper-semicontinuous. Then this mapping is measurable in the sense of Definition 2.1.

A multi-valued mapping $D$ is said to be negatively, strictly, or positively invariant for the MNDS $G$ if

$$
\begin{aligned}
& \subset \\
D\left(\theta_{t} \omega\right) & =G(t, \omega, D(\omega)) \quad \text { for } \omega \in \Omega, t \in \mathbb{R}^{+} .
\end{aligned}
$$

Let $\mathcal{D}$ be the family of multi-valued mappings with values in $C(X)$. We say that a family $K \in \mathcal{D}$ is pullback $D$-attracting if for every $D \in \mathcal{D}$

$$
\lim _{t \rightarrow+\infty} \operatorname{dist}_{X}\left(G\left(t, \theta_{-t} \omega, D\left(\theta_{-t} \omega\right)\right), K(\omega)\right)=0, \text { for all } \omega \in \Omega .
$$

$B \in \mathcal{D}$ is said to be pullback $D$-absorbing if for every $D \in \mathcal{D}$ there exists $T=$ $T(\omega, D)>0$ such that

$$
G\left(t, \theta_{-t} \omega, D\left(\theta_{-t} \omega\right)\right) \subset B(\omega), \text { for all } t \geq T .
$$

The following definition provides the main objective of this article. We have to introduce a particular set system (see Schmalfuß [19]). Let $\mathcal{D}$ be a set of multivalued mappings in $C(X)$ satisfying the inclusion closed property: if we suppose that $D \in \mathcal{D}$ and $D^{\prime}$ is a multi-valued mapping in $C(X)$ such that $D^{\prime}(\omega) \subset D(\omega)$ for $\omega \in \Omega$, then $D^{\prime} \in \mathcal{D}$.

Definition 2.3. A family $\mathcal{A} \in \mathcal{D}$ is said to be a global pullback $\mathcal{D}$-attractor for the MNDS $G$ if it satisfies:

(1) $\mathcal{A}(\omega)$ is compact for any $\omega \in \Omega$;

(2) $\mathcal{A}$ is pullback $\mathcal{D}$-attracting;

(3) $\mathcal{A}$ is negatively invariant.

$\mathcal{A}$ is said to be a strict global pullback $\mathcal{D}$-attractor if the invariance property in the third item is strict.

A natural modification of this definition for MRDS is the following.

Definition 2.4. Suppose that $G$ is an MRDS and suppose that the properties of Definition 2.3 are satisfied. In addition, we suppose that $A$ is a random set with respect to $\mathcal{P}^{c}$. Then $\mathcal{A}$ is called a random global pullback $\mathcal{D}$-attractor.

Now we recall two general results on the existence and uniqueness of pullback and random attractors associated to MNDS and MRDS respectively, which were proved in [6].

THEOREM 2.5. Suppose that the MNDS $G(t, \omega, \cdot)$ is upper-semicontinuous for $t \geq 0$ and $\omega \in \Omega$. Let $K \in \mathcal{D}$ be a multi-valued mapping such that the MNDS is pullback $\mathcal{D}$-asymptotically compact with respect to $K$, i.e. for every sequence $t_{n} \rightarrow+\infty, \omega \in \Omega$ every sequence $y_{n} \in G\left(t_{n}, \theta_{-t_{n}} \omega, K\left(\theta_{-t_{n}} \omega\right)\right)$ is pre-compact. In addition, suppose that $K$ is pullback $\mathcal{D}$-absorbing. Then, the set $\mathcal{A}$ given by

$$
\mathcal{A}(\omega):=\bigcap_{s \geq 0} \overline{\bigcup_{t \geq s} G\left(t, \theta_{-t} \omega, K\left(\theta_{-t} \omega\right)\right)}
$$


is a pullback $\mathcal{D}$-attractor. Furthermore, $\mathcal{A}$ is the unique element from $\mathcal{D}$ with these properties. In addition, if $G$ is a strict $M N D S$, then $\mathcal{A}$ is strictly invariant.

Remark 1. When the property $\mathcal{A} \in \mathcal{D}$ is not satisfied, some difficulties may appear, for example, in proving the strict invariance of the attractor. This is the case if $\mathcal{D}$ is just the set of all bounded subsets of $X$ (see [9]-[10]).

Theorem 2.6. Let $G$ be a MRDS. Under the assumptions in Theorem 2.5, let $\omega \rightarrow G(t, \omega, K(\omega))$ be a random set for $t \geq 0$ with respect to $\mathcal{P}^{c}$. Assume also that $G(t, \omega, K(\omega))$ is closed for all $t \geq 0$ and $\omega \in \Omega$. Then the set $\mathcal{A}$ defined by (2) is a random set with respect to $\mathcal{P}^{c}$, so that it is a random global pullback $\mathcal{D}$-attractor.

\section{Setting of the problem}

Let us consider the lattice stochastic system

$$
\left\{\begin{array}{l}
\frac{d u_{i}}{d t}=\nu\left(u_{i-1}-2 u_{i}+u_{i+1}\right)-h_{i}\left(u_{i}\right)-f_{i}\left(u_{i}\right)+a_{i} \frac{d w_{i}(t)}{d t}, i \in \mathbb{Z} \\
u_{i}(0)=u_{i}^{0}
\end{array}\right.
$$

where $u=\left(u_{i}\right)_{i \in \mathbb{Z}} \in l^{2}, u^{0}=\left(u_{i}^{0}\right)_{i \in \mathbb{Z}} \in l^{2}, \nu>0, a=\left(a_{i}\right)_{i \in \mathbb{Z}} \in l^{2}, w_{i}$ are real independent two-sided Brownian motions (so they satisfy $w_{i}(0)=0$ ) and $f_{i}, h_{i}: \mathbb{R} \rightarrow \mathbb{R}, i \in \mathbb{Z}$, are functions satisfying the following conditions:

$(H 1)$ For all $x \in \mathbb{R}$,

$$
\begin{gathered}
h_{i}(x) x \geq \alpha|x|^{2}-c_{1, i}, \\
\left|h_{i}(x)\right| \leq \beta|x|+c_{2, i},
\end{gathered}
$$

where $c_{1} \in l^{1}, c_{2} \in l^{2}, c_{2, i} \geq 0, \alpha, \beta>0$.

(H2) For all $x \in \mathbb{R}$,

$$
\begin{aligned}
f_{i}(x) x & \geq \gamma|x|^{p}-c_{3, i}, \\
\left|f_{i}(x)\right| & \leq \eta|x|^{p-1}+c_{4, i},
\end{aligned}
$$

where $p \geq 2, c_{3} \in l^{1}, c_{4} \in l^{2}, c_{4, i} \geq 0, \gamma, \eta>0$.

(H3) The maps $h_{i}, f_{i}: \mathbb{R} \rightarrow \mathbb{R}$ are continuous.

We define the operators $A, B, B^{*}: l^{2} \rightarrow l^{2}$ given by

$$
\begin{aligned}
& (B u)_{i}=u_{i+1}-u_{i},\left(B^{*} u\right)_{i}=u_{i-1}-u_{i} \\
& (A u)_{i}=-u_{i-1}+2 u_{i}-u_{i+1}
\end{aligned}
$$

It is easy to see that $A=B^{*} B=B B^{*}$ and $\left(B^{*} u, v\right)=(u, B v)$, for all $u, v \in l^{2}$, where $(\cdot, \cdot)$ denotes the scalar product in $l^{2}$.

Using conditions $(H 1)-(H 2)$ it is not difficult to see that the Nemytski operators $f, h: l^{2} \rightarrow l^{2}$ given by $f(u)=\left(f_{i}\left(u_{i}\right)\right)_{i \in \mathbb{Z}}, h(u)=\left(h_{i}\left(u_{i}\right)\right)_{i \in \mathbb{Z}}$ are well defined. On 
the other hand, we can obtain the following estimates for the norms of $f(u), h(u)$ :

$$
\begin{aligned}
\|h(u)\|_{l^{2}}^{2} & \leq \sum_{i \in \mathbb{Z}}\left(c_{2, i}+\beta\left|u_{i}\right|\right)^{2} \leq 2\left(\left\|c_{2}\right\|_{l^{2}}^{2}+\beta^{2}\|u\|_{l^{2}}^{2}\right) \\
\|f(u)\|_{l^{2}}^{2} & \leq \sum_{i \in \mathbb{Z}}\left(c_{4, i}+\eta\left|u_{i}\right|^{p-1}\right)^{2} \leq 2\left(\left\|c_{4}\right\|_{l^{2}}^{2}+\eta^{2}\|u\|_{l^{2 p-2}}^{2 p-2}\right) \\
& \leq C\left(\left\|c_{4}\right\|_{l^{2}}^{2}+\|u\|_{l^{2}}^{2 p-2}\right) .
\end{aligned}
$$

Moreover, the maps $f, h$ are continuous and weakly continuous.

LEMma 3.1. The maps $f, h: l^{2} \rightarrow l^{2}$ are continuous.

Proof. Let $u^{n} \rightarrow u^{0}$ in $l^{2}$. It is clear that for any $\epsilon>0$ there exist $k_{0}(\epsilon)$ such that

$$
\sum_{|i|>k_{0}}\left|u_{i}^{0}\right|^{2} \leq \epsilon, \sum_{|i|>k_{0}}\left|c_{4, i}\right|^{2} \leq \epsilon, \sum_{|i|>k_{0}}\left|u_{i}^{n}\right|^{2} \leq \epsilon, \forall n
$$

Also, by (H3) we can choose $n_{0}\left(\epsilon, k_{0}\right)$ such that if $n \geq n_{0}$, then

$$
\sum_{|i| \leq k_{0}}\left|f_{i}\left(u_{i}^{n}\right)-f_{i}\left(u_{i}^{0}\right)\right|^{2} \leq \epsilon
$$

Therefore, using (H2) we obtain

$$
\begin{gathered}
\left\|f\left(u^{n}\right)-f\left(u^{0}\right)\right\|^{2}=\sum_{|i| \leq k_{0}}\left|f_{i}\left(u_{i}^{n}\right)-f_{i}\left(u_{i}^{0}\right)\right|^{2}+\sum_{|i|>k_{0}}\left|f_{i}\left(u_{i}^{n}\right)-f_{i}\left(u_{i}^{0}\right)\right|^{2} \\
\leq \epsilon+4 \sum_{|i|>k_{0}}\left(\left|c_{4, i}\right|^{2}+\eta^{2}\left|u_{i}^{n}\right|^{2(p-1)}\right)+4 \sum_{|i|>k_{0}}\left(\left|c_{4, i}\right|^{2}+\eta^{2}\left|u_{i}^{0}\right|^{2(p-1)}\right) \\
\leq 9 \epsilon+4 C_{0} \eta^{2}\left(\sum_{|i|>k_{0}}\left|u_{i}^{n}\right|^{2}+\sum_{|i|>k_{0}}\left|u_{i}^{0}\right|^{2}\right) \leq C_{1} \epsilon
\end{gathered}
$$

where $C_{0}=\sup _{i \in \mathbb{Z}, n \in \mathbb{N} \cup\{0\}}\left|u_{i}^{n}\right|^{2 p-4}$. Thus, $f\left(u^{n}\right) \rightarrow f\left(u^{0}\right)$ in $l^{2}$, which implies the continuity.

For $h$ the proof is similar.

Lemma 3.2. The maps $f, h: l^{2} \rightarrow l^{2}$ are weakly continuous.

Proof. We note that as the space $l^{2}$ endowed with the weak topology satisfies the first axiom of countability, it is not difficult to show that $f$ is weakly continuous if and only if $u_{n} \rightarrow u_{0}$ weakly in $l^{2}$ implies $f\left(u_{n}\right) \rightarrow f\left(u_{0}\right)$.

Take an arbitrary $\xi \in l^{2}$. Then for any $\epsilon>0$ there exists $K_{1}(\epsilon, \xi)$ such that $\sum_{|i| \geq K_{1}}\left|\xi_{i}\right|^{2} \leq \epsilon$. Due to (4), if $\|u\|_{l^{2}} \leq R$, then there exists $M=M(R)$ such that 
$\|f(u)\|_{l^{2}} \leq M$. Thus if $u^{n} \rightarrow u$ weakly in $l^{2}$ we have

$$
\begin{aligned}
\left|\left(f\left(u^{n}\right)-f(u), \xi\right)\right| \leq & \sum_{|i| \leq K_{1}}\left|\left(f_{i}\left(u_{i}^{n}\right)-f_{i}\left(u_{i}\right)\right) \xi_{i}\right| \\
& +\left(\|f(u)\|_{l^{2}}+\left\|f\left(u^{n}\right)\right\|_{l^{2}}\right)\left(\sum_{|i| \geq K_{1}}\left|\xi_{i}\right|^{2}\right)^{\frac{1}{2}} \\
\leq & \|\xi\|_{l^{2}}\left(\sum_{|i| \leq K_{1}}\left|\left(f_{i}\left(u_{i}^{n}\right)-f_{i}\left(u_{i}\right)\right)\right|^{2}\right)^{\frac{1}{2}}+2 M \epsilon,
\end{aligned}
$$

for some $M$. For any $\varepsilon>0$ we choose $\epsilon=\frac{\varepsilon}{4 M}$. Since $u_{i}^{n} \rightarrow u_{i}$ for all $i$ and $f_{i}$ are continuous, we obtain the existence of $N_{1}(\varepsilon, \xi)$ such that $\left(\sum_{|i| \leq K_{1}}\left|\left(f_{i}\left(u_{i}^{n}\right)-f_{i}\left(u_{i}\right)\right)\right|^{2}\right)^{\frac{1}{2}} \leq \frac{\varepsilon}{2\|\xi\|_{l^{2}}}($ for $\xi \neq 0)$. Hence

$$
\left|\left(f\left(u^{n}\right)-f(u), \xi\right)\right| \leq \varepsilon \text { if } n \geq N_{1} .
$$

For $h$ the proof is similar.

We rewrite (3) in the abstract form

$$
\left\{\begin{array}{l}
\frac{d u}{d t}=-\nu A u-h(u)-f(u)+\frac{d W}{d t}, \\
u(0)=u^{0} \in l^{2} .
\end{array}\right.
$$

We will transform equation (5) into a random one by using a suitable change of variable.

First let us describe the probability space associated to the Wiener processes $w_{i}$. Let $W(t, \omega)(W(t)$ for short when no confusion is possible) be the white noise with values in the space $l^{2}$ given by

$$
W(t, \omega)=\left(a_{i} w_{i}(t)\right)_{i \in \mathbb{Z}} \in l^{2}
$$

with the probability space $(\Omega, \mathcal{F}, \mathbb{P})$, where

$$
\Omega=\left\{\omega \in \mathcal{C}\left(\mathbb{R}, l^{2}\right): \omega(0)=0\right\}=C_{0}\left(\mathbb{R}, l^{2}\right) .
$$

$\mathcal{F}$ is the Borel $\sigma$-algebra on $\Omega$ with respect to the compact open topology and $\mathbb{P}$ is the Wiener measure on $\mathcal{F}$. If we define the shift operator

$$
\theta_{t} \omega(\cdot)=\omega(\cdot+t)-\omega(t), \text { for } t \in \mathbb{R}
$$

then we obtain the metric dynamical system $\left(\Omega, \mathcal{F}, \mathbb{P},\left(\theta_{t}\right)_{t \in \mathbb{R}}\right)$.

We consider the filtration on $\Omega$ given by

$$
\mathcal{F}_{s}^{t}=\sigma\left\{W\left(\tau_{2}\right)-W\left(\tau_{1}\right): s \leq \tau_{1} \leq \tau_{2} \leq t\right\},
$$


where $\sigma\left\{W\left(\tau_{2}\right)-W\left(\tau_{1}\right): s \leq \tau_{1} \leq \tau_{2} \leq t\right\}$ is the smallest $\sigma$-algebra generated by $W\left(\tau_{2}\right)-W\left(\tau_{1}\right)$ for all $\tau_{1}, \tau_{2}$ satisfying $s \leq \tau_{1} \leq \tau_{2} \leq t$. We have the property $\theta_{r}^{-1} \mathcal{F}_{s}^{t}=\mathcal{F}_{s+r}^{t+r}$.

The completion of $(\Omega, \mathcal{F}, \mathbb{P})$ will be denoted by $(\Omega, \overline{\mathcal{F}}, \overline{\mathbb{P}})$.

We introduce now an Ornstein-Uhlenbeck process in $l^{2}$ on the metric dynamical system $\left(\Omega, \mathcal{F}, \mathbb{P},\left(\theta_{t}\right)_{t \in \mathbb{R}}\right)$ generated by the Wiener process. Namely, put

$$
z\left(\theta_{t} \omega\right):=-\alpha \int_{-\infty}^{0} e^{\alpha s} \theta_{t} \omega(s) d s
$$

where $\alpha>0$ is taken from condition (H1). This integral makes sense for every path $\omega$ having sub-exponential growth. It is well known that $z$ solves the stochastic differential equation

$$
d z+\alpha z=d W(t)
$$

as $t \rightarrow z\left(\theta_{t} \omega\right)$ is a $\theta_{t}$ invariant stationary solution known as the Ornstein-Uhlenbeck process. This process is adapted with respect to $\mathcal{F}_{-\infty}^{t}$, where

$$
\mathcal{F}_{-\infty}^{t}=\bigvee_{s \leq t} \mathcal{F}_{s}^{t}
$$

Moreover, there exists a $\theta_{t}$-invariant set $\Omega^{\prime} \subset \Omega$ of full measure such that the mapping $s \rightarrow z\left(\theta_{s} \omega\right)$ is continuous for each $\omega \in \Omega^{\prime}$ (so that the process has continuous trajectories) and

$$
\lim _{t \rightarrow+\infty} \frac{\|W(t)\|}{t}=0 \text { for all } \omega \in \Omega^{\prime} .
$$

Also, $z\left(\theta_{t} \omega\right)$ has sub-exponential growth, which means that

$$
\lim _{t \rightarrow \pm \infty} \frac{\log ^{+}\left\|z\left(\theta_{t} \omega\right)\right\|}{t}=0, \text { for } \omega \in \Omega^{\prime} .
$$

See [3] for more details.

Remark 2. In the sequel, all the statements are understood to hold on a $\theta_{t^{-}}$ invariant set $\Omega^{\prime} \subset \Omega$ of full measure, although we shall write for simplicity that they are true for any $\omega \in \Omega$. That is, we keep the notation $\Omega$ for the set $\Omega^{\prime}$.

We now perform the change of variable $v(t)=u(t)-z\left(\theta_{t} \omega\right)$, and problem (5) turns (formally) into

$$
\left\{\begin{array}{l}
\frac{d v}{d t}=-\nu A v-h\left(v+z\left(\theta_{t} \omega\right)\right)-f\left(v+z\left(\theta_{t} \omega\right)\right)+\alpha z\left(\theta_{t} \omega\right)-\nu A z\left(\theta_{t} \omega\right), \\
v(0)=v^{0}=u^{0}-z(\omega)
\end{array}\right.
$$

Let $g^{\omega}: \mathbb{R}^{+} \times l^{2} \rightarrow l^{2}$ be the Nemytski operator given by

$$
g_{i}^{\omega}\left(t, v_{i}\right)=h_{i}\left(v_{i}+z_{i}\left(\theta_{t} \omega\right)\right)+f_{i}\left(v_{i}+z_{i}\left(\theta_{t} \omega\right)\right)-\alpha z_{i}\left(\theta_{t} \omega\right)+\nu\left(A z\left(\theta_{t} \omega\right)\right)_{i}, i \in \mathbb{Z}
$$


From $(H 1)-(H 2)$ we obtain that, for every $x \in \mathbb{R}$,

$$
\begin{aligned}
g_{i}^{\omega}(t, x) x & =h_{i}\left(x+z_{i}\left(\theta_{t} \omega\right)\right) x+f_{i}\left(x+z_{i}\left(\theta_{t} \omega\right)\right) x-\alpha z_{i}\left(\theta_{t} \omega\right) x+\nu\left(A z\left(\theta_{t} \omega\right)\right)_{i} x \\
& \geq \alpha\left|x+z_{i}\left(\theta_{t} \omega\right)\right|^{2}-h_{i}\left(x+z_{i}\left(\theta_{t} \omega\right)\right) z_{i}\left(\theta_{t} \omega\right)-c_{1, i} \\
& +\gamma\left|x+z_{i}\left(\theta_{t} \omega\right)\right|^{p}-f_{i}\left(x+z_{i}\left(\theta_{t} \omega\right)\right) z_{i}\left(\theta_{t} \omega\right)-c_{3, i} \\
& -\alpha z_{i}\left(\theta_{t} \omega\right) x+\nu\left(A z\left(\theta_{t} \omega\right)\right)_{i} x \\
& \geq \alpha\left|x+z_{i}\left(\theta_{t} \omega\right)\right|^{2}-\beta\left(\left|x+z_{i}\left(\theta_{t} \omega\right)\right|+c_{2, i}\right)\left|z_{i}\left(\theta_{t} \omega\right)\right|-c_{1, i} \\
& +\gamma\left|x+z_{i}\left(\theta_{t} \omega\right)\right|^{p}-\eta\left(\left|x+z_{i}\left(\theta_{t} \omega\right)\right|^{p-1}+c_{4, i}\right) z_{i}\left(\theta_{t} \omega\right)-c_{3, i} \\
& -\alpha z_{i}\left(\theta_{t} \omega\right) x+\nu\left(A z\left(\theta_{t} \omega\right)\right)_{i} x .
\end{aligned}
$$

Using Young's inequality and

$$
\alpha\left|x+z_{i}\left(\theta_{t} \omega\right)\right|^{2} \geq \frac{2 \alpha}{3}|x|^{2}-2 \alpha\left|z_{i}\left(\theta_{t} \omega\right)\right|^{2}
$$

we have

$$
\begin{aligned}
g_{i}^{\omega}(t, x) x & \geq \frac{\alpha}{2}|x|^{2}-K\left(\left|z_{i}\left(\theta_{t} \omega\right)\right|^{2}+\left|z_{i}\left(\theta_{t} \omega\right)\right|^{p}+\left|\left(A z\left(\theta_{t} \omega\right)\right)_{i}\right|^{2}\right) \\
& -c_{1, i}-c_{3, i}-c_{2, i}^{2}-c_{4, i}^{2} .
\end{aligned}
$$

On the other hand, and thanks again to $(H 1)-(H 2)$, we obtain

$$
\left|g_{i}^{\omega}(t, x)\right| \leq \beta|x|+\eta|x|^{p-1}+c_{2, i}+c_{4, i}+\alpha\left|z_{i}\left(\theta_{t} \omega\right)\right|+\nu\left|\left(A z\left(\theta_{t} \omega\right)\right)_{i}\right| .
$$

Since $s \rightarrow z\left(\theta_{s} \omega\right)$ is continuous for every $\omega \in \Omega^{\prime}$, it follows from (9)-(10) and the definition of the operator $A$, that the following properties hold:

(G1) For all $x \in \mathbb{R}$,

$$
g_{i}^{\omega}(x) x \geq \lambda|x|^{2}-d_{1, i}(t)
$$

where $\lambda>0, d_{1} \in C\left([0, T], l^{1}\right)$ for any $T>0$.

(G2) For all $x \in \mathbb{R}$,

$$
\left|g_{i}^{\omega}(t, x)\right| \leq C(|x|)|x|+d_{2, i}(t)
$$

where $d_{2} \in C\left([0, T], l^{2}\right)$ for any $T>0, d_{2, i} \geq 0$, and $C(\cdot) \geq 0$ is a continuous increasing function.

(G3) $g_{i}^{\omega}:[0, T] \times \mathbb{R} \rightarrow \mathbb{R}$ are continuous.

Remark 3. Of course, the elements $d_{1}, d_{2}$ depend on $\omega$ but we omit this for simplicity of notation. 
Let $F^{\omega}: \mathbb{R}^{+} \times l^{2} \rightarrow l^{2}$ be defined by $F(v)=-\nu A v-g^{\omega}(t, v)$. Then (8) is rewritten as

$$
\left\{\begin{array}{l}
\frac{d v}{d t}=F^{\omega}(t, v), \\
v(0)=v^{0} .
\end{array}\right.
$$

By (4) and $\|A v\|_{l^{2}} \leq 4\|v\|_{l^{2}}$ we have

$$
\begin{aligned}
\left\|F^{\omega}(t, v)\right\|_{l^{2}} & \leq R_{1}\left(1+\|v\|_{l^{2}}+\left\|v+z\left(\theta_{t} \omega\right)\right\|_{l^{2}}+\left\|v+z\left(\theta_{t} \omega\right)\right\|_{l^{2}}^{p-1}+\left\|z\left(\theta_{t} \omega\right)\right\|_{l^{2}}\right) \\
& \leq R_{2}\left(1+\|v\|_{l^{2}}+\|v\|_{l^{2}}^{p-1}+\left\|z\left(\theta_{t} \omega\right)\right\|_{l^{2}}+\left\|z\left(\theta_{t} \omega\right)\right\|_{l^{2}}^{p-1}\right)
\end{aligned}
$$

From the continuity of the maps $h, f: l^{2} \rightarrow l^{2}$ and $t \rightarrow z\left(\theta_{t} \omega\right)$ we obtain that the map $(t, v) \rightarrow F^{\omega}(t, v)$ is continuous for any $\omega \in \Omega$.

From the weak continuity of the maps $h, f: l^{2} \rightarrow l^{2}$, and the continuity of $t \rightarrow z\left(\theta_{t} \omega\right)$, we obtain that the map $(t, v) \rightarrow F^{\omega}(t, v)$ is weakly continuous for any $\omega \in \Omega$. Hence, by [16, Theorem 2 and Remark 2], for any $v^{0} \in l^{2}$ there exists at least one solution $v(\cdot)=v\left(\cdot, \omega, v^{0}\right) \in C^{1}\left([0, \alpha], l^{2}\right)$ defined on some interval $[0, \alpha]$. The same result can be obtained arguing as in [17]. Also, by standard arguments it can be proved that every solution is defined on the whole interval $[0, T]$, and, moreover, it can be extended to a global one in $[0, \infty)$ (see [17]).

Now, we say that $u(\cdot)=u\left(\cdot, \omega, u^{0}\right)$ is a solution of (5) with initial data $u^{0}$ if $u(t)=v(t)+z\left(\theta_{t} \omega\right)$, where $v(\cdot)$ is a solution of (11) with initial data $v^{0}=$ $u^{0}-z(\omega)$. Hence, our intention is to study the asymptotic behaviour of such generalized solutions of (5).

\section{Existence of the global attractor}

Let $\mathcal{S}\left(v^{0}, \omega\right)$ be the set of all solutions to (11) corresponding to $v^{0} \in l^{2}$ and $\omega \in \Omega$. We define the multi-valued map $G: \mathbb{R}^{+} \times \Omega \times l^{2} \rightarrow P\left(l^{2}\right)$ as follows

$$
G\left(t, \omega, u^{0}\right)=\left\{v(t)+z\left(\theta_{t} \omega\right): v(\cdot) \in \mathcal{S}\left(u^{0}-z(\omega), \omega\right)\right\}
$$

In the sequel let us consider the system $\mathcal{D}$ given by the multi-valued mapping $D$ in $l^{2}$ with $D(\omega) \subset B_{l^{2}}(0, \varrho(\omega))$, the closed ball with center zero and radius $\varrho$, which is supposed to have a sub-exponential growth, i.e.

$$
\lim _{t \rightarrow \pm \infty} \frac{\log ^{+} \varrho\left(\theta_{t} \omega\right)}{t}=0 \quad \text { for } \omega \in \Omega
$$

$\mathcal{D}$ is called the family of sub-exponentially growing multi-functions in $C\left(l^{2}\right)$. Of course, the property on $\mathcal{D}$ given in Definition 2.3 holds.

The following lemma can be proved in a standard way (see e.g. [9, Proposition 4] or [6, Lemma 5.1]).

Lemma 4.1. The map $G$ satisfies $G(0, \omega, \cdot)=\operatorname{id}_{X}$ and $G(t+\tau, \omega, x)=$ $G\left(t, \theta_{\tau} \omega, G(\tau, \omega, x)\right)$ for all $t, \tau \in \mathbb{R}^{+}, x \in l^{2}, \omega \in \Omega$. 


\subsection{The non-autonomous attractor}

In this section we prove the existence of a strict global pullback $\mathcal{D}$-attractor.

We first show the existence of a pullback $\mathcal{D}$-absorbing set $K \in \mathcal{D}$.

LEMMA 4.2. The set defined by

$$
K(\omega)=\left\{y \in l^{2}: y=v+z(\omega) \text { with }\|v\| \leq R(\omega)\right\}
$$

where $R^{2}(\omega)=C \int_{-\infty}^{0} e^{\alpha \tau}\left(\left\|\theta_{\tau} z(\omega)\right\|_{l^{2}}^{2}+\left\|\theta_{\tau} z(\omega)\right\|_{l^{2}}^{p}+1\right) d \tau$, for some $C>0$, is sub-exponentially growing and pullback $\mathcal{D}$-absorbing for $G$.

Proof. Taking the scalar product of (8) with $v$, and using (9) and $l^{2} \subset l^{p}$ we have

$$
\begin{aligned}
\frac{d}{d t}\|v\|_{l^{2}}^{2}+2 \nu(A v, v)+\alpha\|v\|_{l^{2}}^{2} & \leq C_{1}\left(\left\|z\left(\theta_{t} \omega\right)\right\|_{l^{2}}^{2}+\left\|z\left(\theta_{t} \omega\right)\right\|_{l^{p}}^{p}+1\right) \\
& \leq C_{2}\left(\left\|z\left(\theta_{t} \omega\right)\right\|_{l^{2}}^{2}+\left\|z\left(\theta_{t} \omega\right)\right\|_{l^{2}}^{p}+1\right) .
\end{aligned}
$$

By Gronwall's lemma

$$
\left\|v\left(t, \omega, v^{0}\right)\right\|_{l^{2}}^{2} \leq e^{-\alpha t}\left\|v^{0}\right\|_{l^{2}}^{2}+C_{2} \int_{0}^{t} e^{-\alpha(t-s)}\left(\left\|z\left(\theta_{s} \omega\right)\right\|_{l^{2}}^{2}+\left\|z\left(\theta_{s} \omega\right)\right\|_{l^{2}}^{p}+1\right) d s .
$$

Put

$$
R(\omega)=\left(2 C_{2} \int_{-\infty}^{0} e^{\alpha \tau}\left(\left\|\theta_{\tau} z(\omega)\right\|_{l^{2}}^{2}+\left\|\theta_{\tau} z(\omega)\right\|_{l^{2}}^{p}+1\right) d \tau\right)^{\frac{1}{2}}
$$

Since $z\left(\theta_{t} \omega\right)$ has sub-exponential growth, this random variable is well-defined and sub-exponentially growing (this can be shown by arguing as in [3, p.13] or [8, Lemma 4.6]). Also, the ball $K(\omega)$ is pullback $\mathcal{D}$-absorbing for $G$. Indeed, for every $y \in G\left(t, \theta_{-t} \omega, B\left(\theta_{-t} \omega\right)\right)$ there exists a solution $v\left(t, \theta_{-t} \omega, v^{0}\right)$ of (8), with $v^{0}=v^{0}\left(\theta_{-t} \omega\right)=u^{0}\left(\theta_{-t} \omega\right)-z\left(\theta_{-t} \omega\right), u^{0} \in B\left(\theta_{-t} \omega\right)$, such that $y=v\left(t, \theta_{-t} \omega, v^{0}\left(\theta_{-t} \omega\right)\right)+z(\omega)$. We note that $z$ has sub-exponential growth and that $B \in \mathcal{D}$, so that

$$
\lim _{t \rightarrow+\infty}\left\|u^{0}\left(\theta_{-t} \omega\right)-z\left(\theta_{-t} \omega\right)\right\|_{l^{2}}^{2} e^{-\alpha t}=0 \text { for all } \omega \in \Omega
$$

Thus (14) implies the existence of $T(\omega, B)$ such that

$$
\left\|v\left(t, \theta_{-t} \omega, v^{0}\left(\theta_{-t} \omega\right)\right)\right\|_{l^{2}} \leq R(\omega)
$$

and then $y \in K(\omega)$, if $t \geq T(\omega, B)$.

Further we will prove that $G$ is pullback $\mathcal{D}$-asymptotically compact with respect to $K(\omega)$.

Define a smooth function $\theta$ satisfying

$$
\theta(s)=\left\{\begin{array}{l}
0,0 \leq s \leq 1 \\
0 \leq \theta(s) \leq 1,1 \leq s \leq 2 \\
1, s \geq 2
\end{array}\right.
$$


Obviously $\left|\theta^{\prime}(s)\right| \leq C$, for all $s \in \mathbb{R}^{+}$. Put $\rho_{N, i}:=\theta\left(\frac{|i|}{N}\right)$.

First, in the following lemma we establish suitable estimates of the tails of the solutions.

Lemma 4.3. Let $K(\omega)$ be the absorbing set given in (13). Then, for every $\epsilon>0$, there exist $T(\epsilon, \omega)>0$ and $N(\epsilon, \omega)>0$ such that any solution $u(\cdot)$ of (3), given by $u(t)=v(t)+z(\omega)$, with $v(\cdot) \in S\left(u^{0}\left(\theta_{-t} \omega\right)-z\left(\theta_{-t} \omega\right), \theta_{-t} \omega\right)$ and $u_{0}\left(\theta_{-t} \omega\right) \in$ $K\left(\theta_{-t} \omega\right)$, satisfies

$$
\sum_{|i| \geq 2 N(\epsilon, \omega)}\left|u_{i}\left(t, \theta_{-t} \omega, u_{0}\left(\theta_{-t} \omega\right)\right)\right|^{2} \leq \epsilon, \text { for all } t \geq T(\epsilon, \omega) .
$$

Proof. Consider the random equation (8) with $v(t)=u(t)-z\left(\theta_{t} \omega\right)$ and $\left(x_{i}\right)_{i \in \mathbb{Z}}:=$ $\rho_{N, i} v_{i}$. Taking the inner product of (8) with $\left(x_{i}\right)_{i \in \mathbb{Z}}$ in $l^{2}$ we have that

$$
\begin{aligned}
\frac{1}{2} \frac{d}{d t} \sum_{i \in \mathbb{Z}} \rho_{N, i}\left|v_{i}\right|^{2}=- & \nu \sum_{i \in \mathbb{Z}}\left(B v_{i}, B x_{i}\right)-\sum_{i \in \mathbb{Z}} \rho_{N, i} h_{i}\left(v_{i}+z_{i}\left(\theta_{t} \omega\right)\right) v_{i} \\
& -\sum_{i \in \mathbb{Z}} \rho_{N, i} f_{i}\left(v_{i}+z_{i}\left(\theta_{t} \omega\right)\right) v_{i}+\alpha \sum_{i \in \mathbb{Z}} \rho_{N, i} z_{i}\left(\theta_{t} \omega\right) v_{i} \\
& -\nu \sum_{i \in \mathbb{Z}} \rho_{N, i}\left(A z\left(\theta_{t} \omega\right)\right)_{i} v_{i} .
\end{aligned}
$$

Now, we estimate the terms of the right-hand side of (15) in several steps.

Step 1 First, in a similar way as in Lemma 4.1 in [3] we obtain

$$
\sum_{i \in \mathbb{Z}}\left(B v_{i}, B x_{i}\right) \geq \sum_{i \in \mathbb{Z}} \rho_{N, i}\left|v_{i+1}-v_{i}\right|^{2}-\frac{D}{N}\|v\|_{l^{2}}^{2}
$$

where $D=2 C$ and $C$ satisfies $\left|\theta^{\prime}(s)\right| \leq C$.

Step 2 Second, we have

$$
-\sum_{i \in \mathbb{Z}} \rho_{N, i} h_{i}\left(v_{i}+z_{i}\left(\theta_{t} \omega\right)\right) v_{i}=-\sum_{i \in \mathbb{Z}} \rho_{N, i}\left[h_{i}\left(v_{i}+z_{i}\right)\left(v_{i}+z_{i}\right)-h_{i}\left(v_{i}+z_{i}\right) z_{i}\right]
$$

and using conditions in (H1) we obtain that

$$
\begin{aligned}
& -\sum_{i \in \mathbb{Z}} \rho_{N, i} h_{i}\left(v_{i}+z_{i}\left(\theta_{t} \omega\right)\right) v_{i} \\
& \quad \leq \sum_{i \in \mathbb{Z}} \rho_{N, i}\left(c_{1, i}-\alpha\left|v_{i}+z_{i}\right|^{2}\right)+\sum_{i \in \mathbb{Z}} \rho_{N, i}\left(\beta\left|v_{i}+z_{i}\right|\left|z_{i}\right|+c_{2, i}\left|z_{i}\right|\right) .
\end{aligned}
$$


From here, using Hölder's inequality,

$$
\begin{aligned}
&-\sum_{i \in \mathbb{Z}} \rho_{N, i} h_{i}\left(v_{i}+z_{i}\left(\theta_{t} \omega\right)\right) v_{i} \\
& \leq \sum_{i \in \mathbb{Z}} \rho_{N, i}\left|c_{1, i}\right|-\frac{\alpha}{2} \sum_{i \in \mathbb{Z}} \rho_{N, i}\left|v_{i}\right|^{2}+\alpha \sum_{i \in \mathbb{Z}} \rho_{N, i}\left|z_{i}\right|^{2} \\
&+\frac{1}{2} \sum_{i \in \mathbb{Z}} \rho_{N, i}\left|c_{2, i}\right|^{2}+\frac{1}{2} \sum_{i \in \mathbb{Z}} \rho_{N, i}\left|z_{i}\right|^{2}+\frac{\alpha}{4} \sum_{i \in \mathbb{Z}} \rho_{N, i}\left(\left|v_{i}\right|^{2}+\left|z_{i}\right|^{2}\right) \\
&+\frac{2 \beta^{2}}{\alpha} \sum_{i \in \mathbb{Z}} \rho_{N, i}\left|z_{i}\right|^{2}
\end{aligned}
$$

where we have used the inequality $|u|^{2}=|u+s-s|^{2} \leq 2\left(|u+s|^{2}+|s|^{2}\right)$, and whence $|u+s|^{2} \geq \frac{1}{2}|u|^{2}-|s|^{2}$. Then,

$$
\begin{array}{r}
-\sum_{i \in \mathbb{Z}} \rho_{N, i} h_{i}\left(v_{i}+z_{i}\left(\theta_{t} \omega\right)\right) v_{i} \leq \sum_{i \in \mathbb{Z}} \rho_{N, i}\left(\left|c_{1, i}\right|+\left|c_{2, i}\right|^{2}\right)-\frac{\alpha}{4} \sum_{i \in \mathbb{Z}} \rho_{N, i}\left|v_{i}\right|^{2} \\
+\left(\alpha+\frac{1}{2}+\frac{\alpha}{4}+\frac{2 \beta^{2}}{\alpha}\right) \sum_{i \in \mathbb{Z}} \rho_{N, i}\left|z_{i}\right|^{2}
\end{array}
$$

Step 3 In this third step, we estimate the term concerning $f$. Using Young's inequality and the condition (H2) we obtain that

$$
\begin{aligned}
-\sum_{i \in \mathbb{Z}} \rho_{N, i} f_{i}\left(v_{i}+z_{i}\left(\theta_{t} \omega\right)\right) v_{i} \\
=-\sum_{i \in \mathbb{Z}} \rho_{N, i} f_{i}\left(v_{i}+z_{i}\right)\left(v_{i}+z_{i}\right)+\sum_{i \in \mathbb{Z}} \rho_{N, i} f_{i}\left(v_{i}+z_{i}\right) z_{i} \\
\leq \sum_{i \in \mathbb{Z}} \rho_{N, i} c_{3, i}-\gamma \sum_{i \in \mathbb{Z}} \rho_{N, i}\left|v_{i}+z_{i}\right|^{p}+\sum_{i \in \mathbb{Z}} \rho_{N, i}\left[\eta\left|v_{i}+z_{i}\right|^{p-1}+c_{4, i}\right]\left|z_{i}\right| \\
\leq \sum_{i \in \mathbb{Z}} \rho_{N, i} c_{3, i}-\frac{\gamma}{2} \sum_{i \in \mathbb{Z}} \rho_{N, i}\left|v_{i}+z_{i}\right|^{p}+K_{1}(p, \gamma, \eta) \sum_{i \in \mathbb{Z}} \rho_{N, i}\left|z_{i}\right|^{p} \\
\quad+\sum_{i \in \mathbb{Z}} \rho_{N, i}\left|z_{i}\right| c_{4, i} \\
\leq \sum_{i \in \mathbb{Z}} \rho_{N, i}\left(c_{3, i}+c_{4, i}^{2}\right)-\frac{\gamma}{2 C_{p}} \sum_{i \in \mathbb{Z}} \rho_{N, i}\left|v_{i}\right|^{p}+K_{2} \sum_{i \in \mathbb{Z}} \rho_{N, i}\left|z_{i}\right|^{p} \\
\quad+\frac{1}{2} \sum_{i \in \mathbb{Z}} \rho_{N, i}\left|z_{i}\right|^{2}
\end{aligned}
$$

where $K_{2}$ is a constant depending only on $\gamma, p$ and $\eta$.

Step 4 As for the fourth term,

$$
\alpha \sum_{i \in \mathbb{Z}} \rho_{N, i} z_{i}\left(\theta_{t} \omega\right) v_{i} \leq \frac{\alpha}{8} \sum_{i \in \mathbb{Z}} \rho_{N, i}\left|v_{i}\right|^{2}+2 \alpha \sum_{i \in \mathbb{Z}} \rho_{N, i}\left|z_{i}\right|^{2}
$$


Step 5 Finally

$$
\begin{aligned}
& -\nu \sum_{i \in \mathbb{Z}} \rho_{N, i}\left(A z\left(\theta_{t} \omega\right)\right)_{i} v_{i} \\
& \quad=-\nu \sum_{i \in \mathbb{Z}} B z_{i} B\left(\rho_{N, i} v_{i}\right) \\
& \quad=-\nu \sum_{i \in \mathbb{Z}}\left(z_{i+1}-z_{i}\right)\left(\rho_{N, i+1} v_{i+1}-\rho_{N, i} v_{i}\right) \\
& \quad=-\nu \sum_{i \in \mathbb{Z}} \rho_{N, i+1} v_{i+1}\left(z_{i+1}-z_{i}\right)+\nu \sum_{i \in \mathbb{Z}} \rho_{N, i} v_{i}\left(z_{i+1}-z_{i}\right) \\
& \quad \leq \frac{\alpha}{16} \sum_{i \in \mathbb{Z}} \rho_{N, i}\left|v_{i}\right|^{2}+K_{3} \sum_{|i| \geq N-1}\left|z_{i}\right|^{2} .
\end{aligned}
$$

Then, from (16)-(20), and simplifying, we have that

$$
\begin{aligned}
& \frac{d}{d t} \sum_{i \in \mathbb{Z}} \rho_{N, i}\left|v_{i}\right|^{2}+\frac{\alpha}{8} \sum_{i \in \mathbb{Z}} \rho_{N, i}\left|v_{i}\right|^{2} \\
& \leq \frac{2 D \nu}{N}\|v\|_{l^{2}}^{2}+2 \sum_{i \in \mathbb{Z}} \rho_{N, i}\left(\left|c_{1, i}\right|+\left|c_{3, i}\right|+\left|c_{2, i}\right|^{2}+\left|c_{4, i}\right|^{2}\right) \\
& \quad+K_{4} \sum_{|i| \geq N-1}\left|z_{i}\right|^{2}+K_{5} \sum_{|i| \geq N}\left|z_{i}\right|^{p},
\end{aligned}
$$

where $D, K_{4}$ and $K_{5}$ only depend on the parameters of the problem.

We take $N_{0}(\epsilon)$ verifying that

$$
\frac{16}{\alpha} \sum_{i \in \mathbb{Z}} \rho_{N_{0}, i}\left(\left|c_{1, i}\right|+\left|c_{3, i}\right|+\left|c_{2, i}\right|^{2}+\left|c_{4, i}\right|^{2}\right) \leq \frac{\epsilon}{4} .
$$

Thanks to Gronwall's lemma,

$$
\begin{aligned}
\sum_{i \in \mathbb{Z}} \rho_{N, i}\left|v_{i}\left(t, \omega, v_{0}(\omega)\right)\right|^{2} \\
\leq e^{-\frac{\alpha}{8} t} \sum_{i \in \mathbb{Z}} \rho_{N, i}\left|v_{0, i}(\omega)\right|^{2} \\
\quad+\int_{0}^{t} e^{-\frac{\alpha}{8}(t-s)} \frac{2 \nu D}{N}\left\|v\left(s, \omega, v_{0}(\theta)\right)\right\|_{l^{2}}^{2} d s+\frac{\epsilon}{4} \\
\quad+K_{6} \int_{0}^{t} e^{-\frac{\alpha}{8}(t-s)}\left[\sum_{|i| \geq N-1}\left|z_{i}\left(\theta_{s} \omega\right)\right|^{2}+\sum_{|i| \geq N-1}\left|z_{i}\left(\theta_{s} \omega\right)\right|^{p}\right] d s .
\end{aligned}
$$

Now, after replacing $\omega$ by $\theta_{-t} \omega$ we can estimate this expression in several steps.

Step 1 For the first term, using the definition of the absorbing set in Lemma 4.2 and $u_{0}\left(\theta_{-t} \omega\right) \in K\left(\theta_{-t} \omega\right)$, we can find $T_{1}(\epsilon, \omega, K)>0$ such that

$$
e^{-\frac{\alpha}{8} t} \sum_{i \in \mathbb{Z}} \rho_{N, i}\left|v_{0, i}\left(\theta_{-t} \omega\right)\right|^{2} \leq e^{-\frac{\alpha}{8} t} R^{2}\left(\theta_{-t} \omega\right) \leq \frac{\epsilon}{4},
$$


for $t \geq T_{1}$.

Step 2 For the second term, using (14) we have

$$
\begin{aligned}
& \frac{1}{N} \int_{0}^{t} e^{-\frac{\alpha}{8}(t-s)}\left\|v\left(s, \omega, v_{0}\left(\theta_{-t} \omega\right)\right)\right\|_{l^{2}}^{2} d s \\
& \leq \frac{1}{N} \int_{0}^{t} e^{-\frac{\alpha}{8}(t-s)} e^{-\alpha s}\left\|v^{0}\right\|_{l^{2}}^{2} d s \\
& +C_{2} \frac{1}{N} \int_{0}^{t} \int_{0}^{s} e^{-\frac{\alpha}{8}(t-s)} e^{-\alpha(s-r)}\left(\left\|z\left(\theta_{r-t} \omega\right)\right\|_{l^{2}}^{2}+\left\|z\left(\theta_{r-t} \omega\right)\right\|_{l^{2}}^{p}+1\right) d r d s .
\end{aligned}
$$

Then, we estimate the two last integrals. On the one hand,

$$
\frac{1}{N} \int_{0}^{t} e^{-\frac{\alpha}{8}(t-s)} e^{-\alpha s}\left\|v^{0}\left(\theta_{-t} \omega\right)\right\|_{l^{2}}^{2} d s \leq \frac{t}{N}\left\|v^{0}\left(\theta_{-t} \omega\right)\right\|_{l^{2}}^{2} e^{-\frac{\alpha}{8} t}
$$

and, on the other hand, using that $\|z(\omega)\|$ is sub-exponentially growing, and $z\left(\theta_{t} \omega\right)$ is continuous in $t$, we have that

$$
\begin{gathered}
C_{2} \frac{1}{N} \int_{0}^{t} \int_{0}^{s} e^{-\frac{\alpha}{8}(t-s)} e^{-\alpha(s-r)}\left(\left\|z\left(\theta_{r-t} \omega\right)\right\|_{l^{2}}^{2}+\left\|z\left(\theta_{r-t} \omega\right)\right\|_{l^{2}}^{p}+1\right) d r d s \\
\leq C_{2} \frac{1}{N} \int_{0}^{t} \int_{0}^{s} e^{-\frac{\alpha}{8}(t-r)}\left(e^{\frac{\alpha}{16}(t-r)} r(\omega)+1\right) d r d s \\
\leq C_{2} \frac{8^{2}}{\alpha^{2}} \frac{1}{N}(1+4 r(\omega)),
\end{gathered}
$$

where $r(\omega)$ has sub-exponential growth [2, p. 189]. Then, we can find $N_{1}(\epsilon, \omega)$ large enough such that

$$
\frac{1}{N} \int_{0}^{t} e^{-\frac{\alpha}{8}(t-s)}\left\|v\left(s, \omega, v_{0}\left(\theta_{-t} \omega\right)\right)\right\|_{l^{2}}^{2} d s \leq \frac{\epsilon}{8 D \nu}, \text { if } N \geq N_{1} \text {, for all } t \geq 0 .
$$

Step 3 Finally, we estimate the last term in expression (22). For this, we consider an arbitrary $T^{*}>0$, to be determined later on, and we take $t>\max \left\{T_{1}, T^{*}\right\}$. Then

$$
\begin{aligned}
& K_{6} \int_{0}^{t} e^{-\frac{\alpha}{8}(t-s)}\left[\sum_{|i| \geq N-1}\left|z_{i}\left(\theta_{s-t} \omega\right)\right|^{2}+\sum_{|i| \geq N-1}\left|z_{i}\left(\theta_{s-t} \omega\right)\right|^{p}\right] d s \\
& =K_{6} \int_{-t}^{0} e^{\frac{\alpha}{8} s}\left[\sum_{|i| \geq N-1}\left|z_{i}\left(\theta_{s} \omega\right)\right|^{2}+\sum_{|i| \geq N-1}\left|z_{i}\left(\theta_{s} \omega\right)\right|^{p}\right] d s
\end{aligned}
$$

and we split the integration domain in the last integral, $(-t, 0)$, into two others, $\left(-t,-T^{*}\right)$ and $\left(-T^{*}, 0\right)$. Then, we can estimate these new integrals.

Again, using that $\|z(\omega)\|_{l^{2}}$ has sub-exponential growth and $z\left(\theta_{t} \omega\right)$ is continuous in $t$, we have that

$$
K_{6} \int_{-t}^{-T^{*}} e^{\frac{\alpha}{8} s}\left[\left\|z\left(\theta_{s} \omega\right)\right\|_{l^{2}}^{2}+\left\|z_{i}\left(\theta_{s} \omega\right)\right\|_{l^{p}}^{p}\right] d s \leq K_{7} r(\omega) \frac{1}{\alpha} e^{-\frac{\alpha}{16} T^{*}} .
$$


Then, if we take $T^{*} \geq \frac{16}{\alpha} \log \left(\frac{8 r(\omega) K_{7}}{\alpha \epsilon}\right)$ we have that

$$
K_{6} \int_{-t}^{-T^{*}} e^{\frac{\alpha}{8} s}\left[\left\|z\left(\theta_{s} \omega\right)\right\|_{l^{2}}^{2}+\left\|z_{i}\left(\theta_{s} \omega\right)\right\|_{l^{p}}^{p}\right] d s \leq \frac{\epsilon}{8}
$$

As for the other integral we use Lebegue's theorem to find $N_{2}\left(\epsilon, \omega, T^{*}\right)$ such that, if $N \geq N_{2}$,

$$
K_{6} \int_{-T *}^{0} e^{\frac{\alpha}{8} s}\left[\sum_{|i| \geq N-1}\left|z_{i}\left(\theta_{s} \omega\right)\right|^{2}+\sum_{|i| \geq N-1}\left|z_{i}\left(\theta_{s} \omega\right)\right|^{p}\right] d s \leq \frac{\epsilon}{8} .
$$

Then, if we take $\hat{N}>\max \left\{N_{0}, N_{1}, N_{2}\right\}, N \geq \hat{N}$ and $T(\epsilon, \omega)>\max \left\{T_{1}, T^{*}\right\}$, we deduce from (22) that

$$
\sum_{|i|>2 N}\left|v_{i}\left(t, \theta_{-t} \omega, v_{0}\left(\theta_{-t} \omega\right)\right)\right|^{2} \leq \sum_{i \in \mathbb{Z}} \rho_{N, i}\left|v_{i}\left(t, \theta_{-t} \omega, v_{0}\left(\theta_{-t} \omega\right)\right)\right|^{2} \leq \epsilon, \text { if } t \geq T
$$

This implies that

$$
\begin{aligned}
& \sum_{|i| \geq 2 N(\epsilon, \omega)}\left|u_{i}\left(t, \theta_{-t} \omega, u_{0}\left(\theta_{-t} \omega\right)\right)\right|^{2} \\
& \quad \leq 2 \sum_{|i| \geq 2 N(\epsilon, \omega)}\left|v_{i}\left(t, \theta_{-t} \omega, v_{0}\left(\theta_{-t} \omega\right)\right)\right|^{2}+2 \sum_{|i| \geq 2 N(\epsilon, \omega)}\left|z_{i}(\omega)\right|^{2} \\
& \quad \leq 4 \epsilon,
\end{aligned}
$$

for some $N \geq \hat{N}$, if $t \geq T$.

Lemma 4.4. $G$ is pullback $\mathcal{D}$-asymptotically compact with respect to $K \in \mathcal{D}$.

Proof. Let $\omega \in \Omega$. Consider $\left\{t_{n}\right\}_{n \in \mathbb{N}}$ with $\lim _{n \rightarrow \infty} t_{n}=+\infty$ and $\xi^{n} \in$ $G\left(t_{n}, \theta_{-t_{n}} \omega, K\left(\theta_{-t_{n}} \omega\right)\right)$. We can find $x^{n} \in K\left(\theta_{-t_{n}} \omega\right)$ such that

$$
\xi^{n} \in G\left(t_{n}, \theta_{-t_{n}} \omega, x^{n}\right)
$$

We will show that $\left\{\xi^{n}\right\}_{n \in \mathbb{N}}$ possesses a convergent subsequence.

Let $T(\omega, K)$ be the time such that $G\left(t, \theta_{-t} \omega, K\left(\theta_{-t} \omega\right)\right) \subset K(\omega)$ if $t \geq T$. There exists $n_{0}$ verifying that $t_{n} \geq T(\omega)$, for $n \geq n_{0}$. Then $\left\{\xi^{n}\right\} \subset K(\omega)$, so it is weak convergent, that is, there exists $\xi \in l^{2}$ and a subsequence such that

$$
\xi^{n} \rightarrow \xi \text { weakly in } l^{2}
$$

We shall check that this convergence is, in fact, strong.

Let $\epsilon>0$ be arbitrary. Using Lemma 4.3 we obtain the existence of $n_{1}(\epsilon, \omega)$, $M_{0}(\epsilon, \omega)$ such that

$$
\sum_{|i| \geq M_{0}}\left|\xi_{i}^{n}\right|^{2} \leq \epsilon, \text { if } n \geq n_{1}
$$


Also, as $\xi \in l^{2}$, one can find $M_{1}(\epsilon) \geq M_{0}$ such that

$$
\sum_{|i| \geq M}\left|\xi_{i}\right|^{2} \leq \epsilon, \text { if } M \geq M_{1}
$$

We take $M(\epsilon, \omega)=\max \left\{M_{0}, M_{1}\right\}$. The weak convergence (23) ensures that for each $|i| \leq M$,

$$
\xi_{i}^{n} \rightarrow \xi_{i} \text { as } n \rightarrow \infty
$$

From this convergence, there exists $n_{2}(M, \epsilon, \omega) \geq n_{1}$ such that

$$
\sum_{|i| \leq M}\left|\xi_{i}^{n}-\xi_{i}\right|^{2} \leq \epsilon, \text { if } n \geq n_{2}
$$

Then, using these estimates for $n \geq n_{2}$ we have that

$$
\begin{aligned}
\sum_{i \in \mathbb{Z}}\left|\xi_{i}^{n}-\xi_{i}\right|^{2} & \leq \sum_{|i| \leq M}\left|\xi_{i}^{n}-\xi_{i}\right|^{2}+\sum_{|i|>M}\left|\xi_{i}^{n}-\xi_{i}\right|^{2} \\
& \leq \epsilon+2 \sum_{|i| \geq M}\left|\xi_{i}\right|^{2}+2 \sum_{|i| \geq M}\left|\xi_{i}^{n}\right|^{2} \leq 5 \epsilon
\end{aligned}
$$

The proof is therefore finished.

Let us now prove other properties of the cocycle $G$.

Lemma 4.5. Let $v^{0 n}$ be a sequence converging to $v^{0}$ in $l^{2}$ and fix $T>0$. Then, for any $\omega \in \Omega$ and $\epsilon>0$, there exists $K(\epsilon, \omega)$ such that for any solution $v^{n}(\cdot) \in$ $\mathcal{S}\left(v^{0 n}, \omega\right)$ it follows

$$
\sum_{|i| \geq 2 K(\epsilon, \omega)}\left|v_{i}^{n}(t)\right|^{2} \leq \epsilon, \forall t \in[0, T]
$$

Moreover, there exists $v(\cdot) \in \mathcal{S}\left(v^{0}, \omega\right)$ and a subsequence $v^{n_{k}}$ satisfying

$$
v^{n_{k}} \rightarrow v \text { in } C\left([0, T], l^{2}\right)
$$

Proof. For any $\epsilon>0$ there exist $K_{1}(\epsilon), N_{1}(\epsilon)$ such that

$$
\begin{gathered}
\sum_{i \in \mathbb{Z}}\left|v_{i}^{0 n}-v_{i}^{0}\right|^{2}<\frac{\epsilon}{4}, \forall n \geq N_{1}, \\
\sum_{i \in \mathbb{Z}} \rho_{K, i}\left|v_{i}^{0}\right|^{2}<\frac{\epsilon}{4}, \forall K \geq K_{1} .
\end{gathered}
$$

Hence,

$$
\sum_{i \in \mathbb{Z}} \rho_{K, i}\left|v_{i}^{0 n}\right|^{2} \leq 2\left(\sum_{i \in \mathbb{Z}} \rho_{K, i}\left|v_{i}^{0 n}-v_{i}^{0}\right|^{2}+\sum_{i \in \mathbb{Z}} \rho_{K, i}\left|v_{i}^{0}\right|^{2}\right)<\epsilon
$$


if $n \geq N_{1}$ and $K \geq K_{1}$. Obviously, modifying $K_{1}$ appropriately, the result holds true for all $n$. Also, in view of (14), there exists $R_{0}(\omega)>0$ such that

$$
\left\|v^{n}(t)\right\|_{l^{2}} \leq R_{0}(\omega), \forall t \in[0, T], \forall n .
$$

Using inequality (21) and the continuity of $t \rightarrow z\left(\theta_{t} \omega\right)$, one can find $K_{2}(\epsilon, \omega)$ such that

$$
\frac{d}{d t} \sum_{i \in \mathbb{Z}} \rho_{K, i}\left|v_{i}^{n}\right|^{2} \leq \epsilon, \text { if } K \geq K_{2}
$$

Integrating over $(0, t)$ and using $(26)$ we obtain

$$
\sum_{|i| \geq 2 K(\epsilon)}\left|v_{i}^{n}(t)\right|^{2} \leq \sum_{i \in \mathbb{Z}} \rho_{K, i}\left|v_{i}^{n}(t)\right|^{2} \leq \epsilon+T \epsilon
$$

if $K \geq \max \left\{K_{1}, K_{2}\right\}$, so that (24) holds.

Fix now $t \in[0, T]$. In view of (27), passing to a subsequence, we can state that $v^{n}(t) \rightarrow w$ weakly in $l^{2}$. Then, for any $\sigma>0$, there exist $N_{2}(\sigma)$ and $K_{3}(\sigma)$ such that

$$
\begin{aligned}
\left\|v^{n}(t)-w\right\|_{l^{2}}^{2} & \leq \sum_{|i| \leq K_{3}(\sigma)}\left|v_{i}^{n}(t)-w_{i}\right|^{2}+\sum_{|i|>K_{3}(\sigma)}\left|v_{i}^{n}(t)-w_{i}\right|^{2} \\
& \leq \sum_{|i| \leq K_{3}(\sigma)}\left|v_{i}^{n}(t)-w_{i}\right|^{2}+2 \sum_{|i|>K_{3}(\sigma)}\left|v_{i}^{n}(t)\right|^{2}+2 \sum_{|i|>K_{3}(\sigma)}\left|w_{i}(t)\right|^{2} \\
& <\sigma
\end{aligned}
$$

if $n \geq N_{2}$. Hence, $v^{n}(t) \rightarrow w$ strongly in $l^{2}$. It follows that the sequence $v^{n}(t)$ is pre-compact for any $t$. By (12) and (27),

$$
\left\|F^{\omega}\left(t, v^{n}(t)\right)\right\|_{l^{2}}^{2} \leq C(\omega), \forall n \in \mathbb{N}, \forall t \in[0, T],
$$

and from the equality $\frac{d}{d t} v_{i}^{n}=F_{i}^{\omega}\left(v_{i}^{n}\right)$ we obtain that

$$
\left\|\frac{d}{d t} v^{n}(t)\right\|_{l^{2}} \leq C_{1}(\omega)
$$

proving that the sequence $v^{n}$ is equi-continuous. The Ascoli-Arzelà theorem implies the existence of a subsequence $v^{n_{k}}$ converging in $C\left([0, T], l^{2}\right)$ to some function $v(\cdot)$. It is then easy to show that $v$ is a solution of $(11)$. Also, it is clear that $v(0)=v^{0}$.

Lemma 4.5 implies several consequences.

Corollary 4.6. For any $\omega \in \Omega$ and $t \geq 0$ the graph of the map $u^{0} \rightarrow G\left(t, \omega, u^{0}\right)$ is closed. Hence, $G$ has closed values.

Proof. For $\xi^{n} \in G\left(t, \omega, u_{n}^{0}\right)$ there are $v_{n}(\cdot) \in \mathcal{S}\left(u_{n}^{0}-z(\omega), \omega\right)$ such that $\xi^{n}=v_{n}(t)+z\left(\theta_{t} \omega\right)$. Assume that $\xi^{n} \rightarrow \xi$ and $u_{n}^{0} \rightarrow u^{0}$. Applying Lemma 4.5 we obtain, passing to a subsequence, that $v_{n} \rightarrow v$ in $C\left([0, t], l^{2}\right)$, where $v(\cdot) \in \mathcal{S}\left(u^{0}-z(\omega), \omega\right)$. Therefore, $\xi=v(t)+z\left(\theta_{t} \omega\right) \in G\left(t, \omega, u^{0}\right)$. 
Corollary 4.7. $G$ is a strict MNDS.

Proof. It follows from Lemma 4.1 and Corollary 4.6.

Corollary 4.8. For any $\omega \in \Omega$ and $t \geq 0$, the map $G(t, \omega, \cdot)$ has compact values.

Proof. Let $\xi^{n} \in G\left(t, \omega, u^{0}\right)$ be an arbitrary sequence. Take $v_{n}(\cdot) \in$ $\mathcal{S}\left(u^{0}-z(\omega), \omega\right)$ such that $\xi^{n}=v_{n}(t)+z\left(\theta_{t} \omega\right)$. By Lemma 4.5 there exists $v(\cdot) \in \mathcal{S}\left(u^{0}-z(\omega), \omega\right)$ satisfying $v_{n_{k}} \rightarrow v$ in $C\left([0, t], l^{2}\right)$ for some subsequence. Hence, $\xi^{n_{k}}=v_{n_{k}}(t)+z\left(\theta_{t} \omega\right) \rightarrow v(t)+z\left(\theta_{t} \omega\right) \in G\left(t, \omega, u^{0}\right)$ in $l^{2}$.

Proposition 4.9. For any $\omega \in \Omega$ and $t \geq 0$, the map $u^{0} \rightarrow G\left(t, \omega, u^{0}\right)$ is upper semi-continuous.

Proof. Suppose the opposite. Then there exist $u^{0}, t>0$, a neighborhood $O$ of $G\left(t, \omega, u^{0}\right)$ and sequences $u_{n}^{0} \rightarrow u^{0}, \xi^{n} \in G\left(t, \omega, u_{n}^{0}\right)$ such that $\xi^{n} \notin O$. Let $\xi^{n}=v_{n}(t)+z\left(\theta_{t} \omega\right)$, where $v_{n}(\cdot) \in \mathcal{S}\left(u_{n}^{0}-z(\omega), \omega\right)$. By Lemma 4.5 we obtain that, up to a subsequence, $v_{n} \rightarrow v$ in $C\left([0, t], l^{2}\right)$, where $v(\cdot) \in \mathcal{S}\left(u^{0}-z(\omega), \omega\right)$. Thus, $\xi^{n} \rightarrow v(t)+z\left(\theta_{t} \omega\right) \in G\left(t, \omega, u^{0}\right)$ in $l^{2}$, which is a contradiction.

Finally, we have the following result.

TheOREM 4.10. The MNDS G possesses a unique $\mathcal{D}$-pullback global strictly invariant attractor $\mathcal{A}(\omega)$, defined by (2), where $K(\omega)$ is the set given in (13).

Proof. This theorem follows from Theorem 2.5 using Corollary 4.7, Lemmas 4.2, 4.4 and Proposition 4.9.

\subsection{The random attractor}

In order to obtain that $\mathcal{A}(\omega)$ is a random pullback $\mathcal{D}$-attractor we need to check that it is a random set. And, to prove the measurability of the global attractor $\mathcal{A}(\omega)$ we need to obtain some properties concerning the map $\omega \rightarrow G(t, \omega, K(\omega))$, where $K(\omega) \in \mathcal{D}$ is the pullback $\mathcal{D}$-absorbing set given in Lemma 4.2. Also, we have to prove that $G$ is an MRDS.

For $N \in \mathbb{N}$, we consider the sets

$$
\Omega_{N}:=\left\{\omega \in \Omega:\|\omega(t)\|_{l^{2}} \leq N e^{\zeta|t|}, \text { for } t \in \mathbb{R}\right\}
$$

where $0<p \zeta<\alpha$. Then it is not difficult to check that $\Omega=\cup_{N} \Omega_{N}$ and $\Omega_{N} \in \mathcal{F}$. It is shown in [7] that $\Omega_{N}$ is a polish space for any $N$.

We need the continuity of the map $\mathbb{R} \times \Omega_{N} \ni(t, \omega) \rightarrow z\left(\theta_{t} \omega\right)$.

Lemma 4.11. For any $N \in \mathbb{N}$ there exists $M(N)$ such that

$$
\left\|z\left(\theta_{t} \omega\right)\right\|_{l^{2}} \leq M e^{\zeta|t|}
$$

for all $\omega \in \Omega_{N}$. 
Proof. We have

$$
\begin{aligned}
\left\|z\left(\theta_{t} \omega\right)\right\|_{l^{2}} & \leq \alpha \int_{-\infty}^{0} e^{\alpha s}\left\|\theta_{t} \omega(s)\right\| d s \leq \alpha \int_{-\infty}^{0} e^{\alpha s}\|\omega(t+s)\| d s+\|\omega(t)\| \\
& \leq \alpha N \int_{-\infty}^{0} e^{\alpha s} e^{\zeta|t+s|} d s+N e^{\zeta|t|} \leq N e^{\zeta|t|}\left(1+\alpha \int_{-\infty}^{0} e^{(\alpha-\zeta) s}\right) \\
& \leq N\left(1+\frac{\alpha}{\alpha-\zeta}\right) e^{\zeta|t|} .
\end{aligned}
$$

Lemma 4.12. The map $\mathbb{R} \times \Omega_{N} \ni(t, \omega) \rightarrow z\left(\theta_{t} \omega\right)$ is continuous.

Proof. Let $t_{n} \rightarrow t_{0}, \omega_{n} \rightarrow \omega_{0}$. Then

$$
\begin{gathered}
\left\|z\left(\theta_{t_{n}} \omega_{n}\right)-z\left(\theta_{t_{0}} \omega_{0}\right)\right\|_{l^{2}}=\left\|\alpha \int_{-\infty}^{0} e^{\alpha s} \theta_{t_{n}} \omega_{n}(s) d s-\alpha \int_{-\infty}^{0} e^{\alpha s} \theta_{t_{0}} \omega_{0}(s) d s\right\|_{l^{2}} \\
\leq \alpha \int_{-\infty}^{0} e^{\alpha s}\left\|\theta_{t_{n}} \omega_{n}(s)-\theta_{t_{0}} \omega_{0}(s)\right\|_{l^{2}} d s \\
\leq \alpha \int_{-\infty}^{0} e^{\alpha s}\left\|\omega_{n}\left(s+t_{n}\right)-\omega_{0}\left(s+t_{0}\right)\right\|_{l^{2}} d s+\left\|\omega_{n}\left(t_{n}\right)-\omega_{0}\left(t_{0}\right)\right\|_{l^{2}} .
\end{gathered}
$$

Due to the definition of $\Omega_{N}$ we have $\left\|\omega_{n}(\tau)-\omega_{0}(\bar{\tau})\right\|_{l^{2}} \leq\left\|\omega_{n}(\tau)\right\|_{l^{2}}+$ $\left\|\omega_{0}(\bar{\tau})\right\|_{l^{2}} \leq N\left(e^{\zeta|\tau|}+e^{\zeta|\bar{\tau}|}\right)$. Thus, for any $\varepsilon>0$, there exists $T(\varepsilon)>t_{0}$ (and then, without loss of generality we can assume that $T(\varepsilon)>t_{n}$ also) such that

$$
\alpha \int_{-\infty}^{-T} e^{\alpha s}\left\|\omega_{n}\left(s+t_{n}\right)-\omega_{0}\left(s+t_{0}\right)\right\|_{l^{2}} d s \leq 2 \alpha N e^{\zeta|\bar{t}|} \int_{-\infty}^{-T} e^{(\alpha-\zeta) s} d s<\frac{\varepsilon}{3}
$$

where $\left|t_{0}\right|<|\bar{t}|$ (and thus, again, we can assume $\left|t_{n}\right|<|\bar{t}|$ ). Thus, if we take $n_{0}(\varepsilon, T(\varepsilon))$ such that $\left\|\omega_{n}\left(s+t_{n}\right)-\omega_{0}\left(s+t_{0}\right)\right\|_{l^{2}}<\varepsilon / 3$, for any $s \in[-T, 0]$ and $n \geq n_{0}$, then we get

$$
\left\|z\left(\theta_{t_{n}} \omega_{n}\right)-z\left(\theta_{t_{0}} \omega_{0}\right)\right\|_{l^{2}}<\varepsilon, \text { if } n \geq n_{0} .
$$

Let $\mathcal{F}_{\Omega_{N}}$ be the trace $\sigma$-algebra of $\mathcal{F}$ with respect to $\Omega_{N}$ and let $B_{\Omega_{N}}(a, r), a \in$ $\Omega_{N}, r>0$ be a ball in $\Omega_{N}$. These balls can be generated by $B_{\Omega}(a, r) \cap \Omega_{N}$ where $B_{\Omega}(a, r)$ is a ball in $\Omega$. The same is true for all open sets in $\Omega_{N}$. Hence $\mathcal{F}_{\Omega_{N}}$ is just the Borel- $\sigma$-algebra of $\Omega_{N}$. Moreover, since $\Omega_{N} \in \mathcal{F}$ we have $\mathcal{F}_{\Omega_{N}} \subset \mathcal{F}$.

Let us define

$$
\mathbb{P}_{\Omega_{N}}(A):=\mathbb{P}(A), \text { for } A \in \mathcal{F}_{\Omega_{N}}
$$


that is, $\mathbb{P}_{\Omega_{N}}$ is just the restriction of $\mathbb{P}$ to $\mathcal{F}_{\Omega_{N}}$. Also, let $\overline{\mathcal{F}}_{\Omega_{N}}$ be the completion of $\mathcal{F}_{\Omega_{N}}$ with respect to $\mathbb{P}_{\Omega_{N}}$.

The following facts are proved in [7]:

(1) $\mathbb{P}_{\Omega_{N}}$ is a finite measure on $\left(\Omega_{N}, \mathcal{F}_{\Omega_{N}}\right)$.

(2) If $A \in \overline{\mathcal{F}}_{\Omega_{N}}$, then $A \in \overline{\mathcal{F}}$.

Now we establish the continuity of the random radius $R(\omega)$ given in Lemma 4.2 over $\Omega_{N}$.

Lemma 4.13. The map $\omega \rightarrow R(\omega)$ is continuous on $\Omega_{N}$.

Proof. Let $\omega_{n} \rightarrow \omega_{0}$ in $\Omega_{N}$. By Lemma 4.12 we have

$$
e^{\alpha \tau}\left(\left\|\theta_{\tau} z\left(\omega_{n}\right)\right\|_{l^{2}}^{2}+\left\|\theta_{\tau} z\left(\omega_{n}\right)\right\|_{l^{2}}^{p}+1\right) \rightarrow e^{\alpha \tau}\left(\left\|\theta_{\tau} z\left(\omega_{0}\right)\right\|_{l^{2}}^{2}+\left\|\theta_{\tau} z\left(\omega_{0}\right)\right\|_{l^{2}}^{p}+1\right),
$$

as $n \rightarrow \infty$. On the other hand, by Lemma 4.11 we obtain the majorant

$$
e^{\alpha \tau}\left(\left\|\theta_{\tau} z\left(\omega_{n}\right)\right\|_{l^{2}}^{2}+\left\|\theta_{\tau} z\left(\omega_{n}\right)\right\|_{l^{2}}^{p}+1\right) \leq e^{\alpha \tau}+M^{2} e^{(\alpha-2 \zeta) \tau}+M^{p} e^{(\alpha-p \zeta) \tau},
$$

which is integrable due to the restriction $p \zeta<\alpha$.

The result follows from Lebesgue's theorem.

Concerning $\Omega_{N}$ we can obtain stronger properties for the cocycle $G$.

Lemma 4.14. The map $\mathbb{R}^{+} \times \Omega_{N} \times l^{2} \ni\left(t, \omega, u^{0}\right) \rightarrow G\left(t, \omega, u^{0}\right)$ is upper semicontinuous.

Proof. If this is not true, then there exist $u^{0}, t_{0}>0, \omega_{0} \in \Omega_{N}$, a neighborhood $O$ of $G\left(t_{0}, \omega_{0}, u^{0}\right)$ and sequences $t_{n} \rightarrow t_{0}, \omega_{n} \rightarrow \omega_{0}$ in $\Omega_{N}, u_{n}^{0} \rightarrow u^{0}$ in $l^{2}, \xi^{n} \in$ $G\left(t_{n}, \omega_{n}, u_{n}^{0}\right)$ such that $\xi^{n} \notin O$. We will prove that, up to a subsequence, $\xi^{n} \rightarrow$ $\xi \in G\left(t_{0}, \omega_{0}, u^{0}\right)$, which is a contradiction.

Let $v^{n}(\cdot) \in \mathcal{S}\left(v^{0 n}, \omega_{n}\right)$ be such that $\xi^{n}=v^{n}\left(t_{n}\right)+z\left(\theta_{t_{n}} \omega_{n}\right)$, where $v^{0 n}=$ $u^{0 n}-z\left(\omega_{n}\right)$. By Lemma 4.12 we have that $z\left(\theta_{t_{n}} \omega_{n}\right) \rightarrow z\left(\theta_{t_{0}} \omega_{0}\right)$ and $v^{0 n} \rightarrow v^{0}=$ $u^{0}-z\left(\omega_{0}\right)$.

Due to these properties and Lemma 4.13, one can state that (26) and (27) hold in the same way as in Lemma 4.5, where $T>t_{n}, T>t_{0}$, and $R_{0}$ is a common constant for any $\omega_{n}$.

Lemma 4.12 and the continuity of $t \rightarrow z\left(\theta_{t} \omega_{0}\right)$ imply that $z\left(\theta_{t} \omega_{n}\right) \rightarrow z\left(\theta_{t} \omega_{0}\right)$ in $C\left([0, T], l^{2}\right)$. Hence, arguing as in the proof of Lemma 4.5, one can prove that, for any $\epsilon>0$, there exist $K_{2}(\epsilon), N_{2}(\epsilon)$ such that $\sum_{i \in \mathbb{Z}} \rho_{K, i}\left|z_{i}\left(\theta_{t} \omega_{n}\right)\right|^{2}<\epsilon$, if $K \geq K_{2}, n \geq N_{2}$. Then, using inequalities (21) and (27), we can find $K_{3}(\epsilon)$ such that

$$
\frac{d}{d t} \sum_{i \in \mathbb{Z}} \rho_{K, i}\left|v_{i}^{n}\right|^{2} \leq \epsilon, \text { if } K \geq K_{3}
$$

Similarly to the proof of Lemma 4.5 we obtain that the sequence $v^{n}(t)$ is pre-compact for any $t$. By (12), (27) and the fact that $z\left(\theta_{t} \omega_{n}\right) \rightarrow z\left(\theta_{t} \omega_{0}\right)$ in $C\left([0, T], l^{2}\right)$, we obtain

$$
\left\|F^{\omega_{n}}\left(t, v^{n}(t)\right)\right\|_{l^{2}}^{2} \leq C, \forall n \in \mathbb{N}, \forall t \in[0, T],
$$


and the equality $\frac{d}{d t} v_{i}^{n}=F_{i}^{\omega_{n}}\left(t, v_{i}^{n}\right)$ implies

$$
\left\|\frac{d}{d t} v^{n}(t)\right\|_{l^{2}} \leq C_{1}
$$

which proves that the sequence $v^{n}$ is equi-continuous. The Ascoli-Arzelà theorem implies then the existence of a subsequence $v^{n_{k}}$ converging to some function $v(\cdot)$ in $C\left([0, T], l^{2}\right)$.

From the continuity of the maps $h, f: l^{2} \rightarrow l^{2}$ and $(t, \omega) \rightarrow z\left(\theta_{t} \omega\right)$ it follows that the map $(\omega, t, v) \rightarrow F^{\omega}(t, v)$ is continuous. Thus, it is easy to show that $v$ is a solution of (11). Also, it is clear that $v(0)=v^{0}$.

It follows that $\xi^{n_{k}} \rightarrow \xi=v\left(t_{0}\right)+z\left(\theta_{t_{0}} \omega_{0}\right) \in G\left(t, \omega_{0}, u^{0}\right)$.

Now, the following result is a consequence of Lemma 2.2.

Corollary 4.15. The map $\left(t, \omega, u^{0}\right) \rightarrow G\left(t, \omega, u^{0}\right)$ is $\mathcal{B}\left(\mathbb{R}^{+}\right) \otimes \mathcal{F}_{\Omega_{N}} \otimes \mathcal{B}\left(l^{2}\right)$ measurable.

Further, we need some properties of the map $\Omega_{N} \ni \omega \rightarrow G(t, \omega, K(\omega))$.

Lemma 4.16. Let $v^{0 n} \rightarrow v^{0}$ weakly in $l^{2}, \omega_{n} \rightarrow \omega_{0}$ in $\Omega_{N}$ and fix $T>0$. Then there exist $v(\cdot) \in \mathcal{S}\left(v^{0}, \omega_{0}\right)$ and a subsequence $v^{n_{k}} \in \mathcal{S}\left(v^{0 n_{k}}, \omega_{n_{k}}\right)$ such that

$$
\begin{aligned}
v^{n_{k}} & \rightarrow v \text { weakly in } L^{2}\left(0, T ; l^{2}\right), \\
v^{n_{k}}(t) & \rightarrow v(t) \text { weakly in } l^{2} \text { for all } t \in[0, T] .
\end{aligned}
$$

Proof. At light of (14) and Lemma 4.13 there exists $R_{0}>0$ such that

$$
\left\|v^{n}(t)\right\|_{l^{2}} \leq R_{0}, \forall t \in[0, T], \forall n .
$$

Thus, by (12) and the convergence $z\left(\theta_{t} \omega_{n}\right) \rightarrow z\left(\theta_{t} \omega_{0}\right)$ in $C\left([0, T], l^{2}\right)$,

$$
\left\|F^{\omega_{n}}\left(t, v^{n}(t)\right)\right\|_{l^{2}} \leq C, \forall n \in \mathbb{N}, \forall t \in[0, T],
$$

and from the equality $\frac{d}{d t} v_{i}^{n}=F_{i}^{\omega_{n}}\left(v_{i}^{n}\right)$ we obtain that

$$
\left\|\frac{d}{d t} v^{n}(t)\right\|_{l^{2}} \leq C
$$

Hence, there exist $v, \chi \in L^{2}\left(0, T ; l^{2}\right)$ such that, up to a subsequence,

$$
v^{n} \rightarrow v, \frac{d}{d t} v^{n} \rightarrow \frac{d}{d t} v, F^{\omega_{n}}\left(\cdot, v^{n}(\cdot)\right) \rightarrow \chi \text { weakly in } v \in L^{2}\left(0, T ; l^{2}\right) .
$$

Also, for any $\xi \in l^{2}$ we have

$$
\left(v^{n}(t), \xi\right)=\left(v^{0 n}, \xi\right)+\int_{0}^{t}\left(\frac{d v^{n}}{d \tau}, \xi\right) d \tau \rightarrow\left(v^{0}, \xi\right)+\int_{0}^{t}\left(\frac{d v}{d \tau}, \xi\right) d \tau=(v(t), \xi)
$$


where the last equality follows from $v, \frac{d v}{d t} \in L^{2}\left(0, T ; l^{2}\right)$. Hence,

$$
v^{n}(t) \rightarrow v(t) \text { weakly in } l^{2} \text { for all } t \in[0, T] .
$$

It follows from the weakly continuity of the maps $h, f: l^{2} \rightarrow l^{2}$ and the continuity of $(t, \omega) \rightarrow z\left(\theta_{t} \omega\right)$ that

$$
F^{\omega_{n}}\left(t, v^{n}(t)\right) \rightarrow F^{\omega_{0}}(t, v(t)) \text { weakly in } l^{2} \text { for all } t \in[0, T] .
$$

Also by (30) we get

$$
\left|\left(F^{\omega_{n}}\left(t, v^{n}(t)\right), \xi\right)\right| \leq C\|\xi\|_{l^{2}}, \forall n \in \mathbb{N}, \forall t \in[0, T],
$$

and then Lebesgue's theorem gives

$$
\left(F^{\omega_{n}}\left(t, v^{n}(t)\right), \xi\right) \rightarrow\left(F^{\omega_{0}}(t, v(t)), \xi\right) \text { in } L^{1}(0, T) \text { for all } \xi \in l^{2} .
$$

Thus,

$$
F^{\omega_{0}}(\cdot, v(\cdot))=\chi
$$

and

$$
v(t)=v^{0}+\int_{0}^{t} \frac{d v}{d \tau} d \tau=v^{0}+\int_{0}^{t} F^{\omega_{0}}(\tau, v(\tau)) d \tau, \text { for all } t \in[0, T]
$$

which implies that $v(\cdot) \in \mathcal{S}\left(v^{0}, \omega_{0}\right)$.

Lemma 4.17. The map $\Omega_{N} \ni \omega \rightarrow G(t, \omega, K(\omega))$ is $\overline{\mathcal{F}}_{\Omega_{N}}$-measurable for any $t \geq 0$. Also, $G(t, \omega, K(\omega))$ is closed for all $t \geq 0, \omega \in \Omega_{N}$.

Proof. It is well known [12, Chapter III] that $D(\omega)$ is a random set with respect to $\mathcal{P}^{c}$ if and only if the graph of $D(\omega)$, given by

$$
\operatorname{Gr}(D):=\left\{(\omega, x) \in \Omega_{N} \times l^{2}: x \in D(\omega)\right\}
$$

belongs to $\overline{\mathcal{F}}_{\Omega_{N}} \otimes \mathcal{B}\left(l^{2}\right)$. Hence to prove the first statement it is sufficient to show that the graph of the map $\omega \rightarrow G(t, \omega, K(\omega))$ belongs to $\overline{\mathcal{F}}_{\Omega_{N}} \otimes \mathcal{B}\left(l^{2}\right)$, and this is true if the graph is closed.

Let $\omega \rightarrow \omega_{0}$ in $\Omega_{N}$ and $\xi^{n} \rightarrow \xi$ in $l^{2}$, where $\xi^{n} \in G\left(t, \omega_{n}, u^{0 n}\right)$ and $u^{0 n} \in K\left(\omega_{n}\right)$. We have to show that $\xi \in G\left(t, \omega_{0}, K\left(\omega_{0}\right)\right)$. Take $v_{n}(\cdot) \in \mathcal{S}\left(u^{0 n}-z\left(\omega_{n}\right), \omega_{n}\right)$ such that $\xi^{n}=v_{n}(t)+z\left(\theta_{t} \omega_{n}\right)$.

By the definition of $K(\omega)$ we have that $v^{n 0}=u^{0 n}-z\left(\omega_{n}\right)$ satisfies $\left\|v^{0 n}\right\|_{l^{2}} \leq$ $R\left(\omega_{n}\right)$. Then by Lemmas $4.12,4.13$ we obtain, passing to a subsequence, that $v^{n 0} \rightarrow v^{0}$ weakly in $l^{2}$, where $\left\|v^{0}\right\|_{l^{2}} \leq R\left(\omega_{0}\right)$, and $u^{0 n} \rightarrow u^{0}=v^{0}+z\left(\omega_{0}\right) \in K\left(\omega_{0}\right)$ weakly in $l^{2}$.

In view of Lemma 4.16 there exists $v(\cdot) \in \mathcal{S}\left(v^{0}, \omega_{0}\right)$ and a subsequence such that

$$
v^{n}(t) \rightarrow v(t) \text { weakly in } l^{2} \text { for all } t \in[0, T]
$$


Thus, $\xi^{n}=v_{n}(t)+z\left(\theta_{t} \omega_{n}\right) \rightarrow v(t)+z\left(\theta_{t} \omega_{0}\right)$ weakly in $l^{2}$, so that $\xi=v(t)+$ $z\left(\theta_{t} \omega_{0}\right) \in G\left(t, \omega_{0}, u_{0}\right) \subset G\left(t, \omega_{0}, K\left(\omega_{0}\right)\right)$.

Since the graph is closed, it is obvious that $G(t, \omega, K(\omega))$ is closed for all $t \geq 0$, $\omega \in \Omega_{N}$.

Theorem 4.18. The MNDS G is an MRDS. Also, the pullback $\mathcal{D}$-attractor $\mathcal{A}(\omega)$ given in Theorem 4.10 is a random set with respect to $\overline{\mathcal{F}}_{\Omega}$, and then it is the unique random global pullback $\mathcal{D}$-attractor for $G$.

Proof. Let us prove that $G$ is an MRDS. As $G$ is an MNDS, it remains to show that the map $(t, \omega, x) \rightarrow G(t, \omega, x)$ is $\mathcal{B}\left(\mathbb{R}^{+}\right) \otimes \mathcal{F} \otimes \mathcal{B}(X)$ measurable. Let $\mathcal{O}$ be an open set of $l^{2}$. Then, by Corollary 4.15, we have that the set

$$
\left\{(t, \omega, x) \in \mathbb{R}^{+} \times \Omega_{N} \times l^{2}: G(t, \omega, x) \cap \mathcal{O} \neq \emptyset\right\}:=A_{N, \mathcal{O}}
$$

belongs to $\mathcal{B}\left(\mathbb{R}^{+}\right) \otimes \mathcal{F}_{\Omega_{N}} \otimes \mathcal{B}\left(l^{2}\right)$, so that $A_{N, \mathcal{O}} \in \mathcal{B}\left(\mathbb{R}^{+}\right) \otimes \mathcal{F} \otimes \mathcal{B}\left(l^{2}\right)$. Hence

$$
\begin{aligned}
& \left\{(t, \omega, x) \in \mathbb{R}^{+} \times \Omega \times l^{2}: G(t, \omega, x) \cap \mathcal{O} \neq \emptyset\right\} \\
& =\bigcup_{N=1}^{\infty}\left\{(t, \omega, x) \in \mathbb{R}^{+} \times \Omega_{N} \times l^{2}: G(t, \omega, x) \cap \mathcal{O} \neq \emptyset\right\} \\
& =\bigcup_{N=1}^{\infty} A_{N, \mathcal{O}} \in \mathcal{B}\left(\mathbb{R}^{+}\right) \otimes \mathcal{F} \otimes \mathcal{B}\left(l^{2}\right),
\end{aligned}
$$

and then $G$ is an MRDS.

Furthermore, in view of Lemma 4.17, the map $\Omega_{N} \ni \omega \rightarrow G(t, \omega, K(\omega))$ is $\overline{\mathcal{F}}_{\Omega_{N}}$-measurable for any $t \geq 0$. Hence, for a fixed $t \geq 0$, the set

$$
\left\{\omega \in \Omega_{N}: G(t, \omega, K(\omega)) \cap \mathcal{O} \neq \emptyset\right\}:=C_{N, \mathcal{O}}
$$

belongs to $\overline{\mathcal{F}}_{\Omega_{N}}$, so $C_{N, \mathcal{O}} \in \overline{\mathcal{F}}_{\Omega}$ and then

$$
\{\omega \in \Omega: G(t, \omega, K(\omega)) \cap \mathcal{O} \neq \emptyset\}=\bigcup_{N=1}^{\infty} C_{N, \mathcal{O}} \in \overline{\mathcal{F}}_{\Omega} .
$$

Due to Theorem 2.6 the pullback $\mathcal{D}$-attractor $\mathcal{A}(\omega)$ is a random set with respect to $\overline{\mathcal{F}}_{\Omega}$, and then it is the unique random global pullback $\mathcal{D}$-attractor for $G$.

\section{Acknowledgements}

This work was partially supported by Spanish Ministerio de Ciencia e Innovación, Projects MTM2008-00088 and MTM2009-11820, the Consejería de Innovación, Ciencia y Empresa (Junta de Andalucía), Proyecto de Excelencia P07FQM-02468, and the Consejería de Cultura y Educación (Comunidad Autónoma de Murcia), grant 08667/PI/08.

\section{References}

[1] A.Y. Abdallah, Exponential attractors for first-order lattice dynamical systems, J. Math. Anal. Appl., 339 (2008), 217-224. 
[2] L. Arnold, "Random dynamical systems", Springer-Verlag, Berlin, 1998.

[3] P.W. Bates, H. Lisei, K. Lu, Attractors for stochastic lattice dynamical systems, Stoch. Dyn., 6 (2006), $1-21$.

[4] P.W. Bates, K. Lu \& B. Wang, Attractors for lattice dynamical systems, Internat. J. Bifur. Chaos, 11 (2001), 143-153.

[5] W.J. Beyn, S.Yu. Pilyugin, Attractors of Reaction Diffusion Systems on Infinite Lattices, J. Dynam. Differential Equations, 15 (2003), 485-515.

[6] T. Caraballo, M.J. Garrido-Atienza, B. Schmalfuß, J. Valero, Non-autonomous and random attractors for delay random semilinear equations without uniqueness, Discrete Contin. Dyn. Syst., 21 (2008), 415-443.

[7] T. Caraballo, M.J. Garrido-Atienza, B. Schmalfuß, J. Valero, Asymptotic behaviour of a stochastic semilinear dissipative functional equation without uniqueness of solutions, submitted.

[8] T. Caraballo, P.E. Kloeden, B. Schmalfuß, Exponentially stable stationary solutions for stochastic evolution equations and their perturbation, Appl. Math. Optim., 50 (2004), 183-207.

[9] T. Caraballo, J. A. Langa, J. Valero, Global attractors for multivalued random dynamical systems, Nonlinear Anal., 48 (2002), 805-829.

[10] T. Caraballo, J. A. Langa, and J. Valero, Global attractors for multivalued random dynamical systems generated by random differential inclusions with multiplicative noise, J. Math. Anal. Appl., 260 (2001), 602-622.

[11] T. Caraballo, K. Lu, Attractors for stochastic lattice dynamical systems with a multiplicative noise, Front. Math. China, 3 (2008), 317-335.

[12] C. Castaing, M. Valadier, "Convex Analysis and Measurable Multifunctions", Lecture Notes in Mathematics, Vol. 580, Springer-Verlag, Berlin, 1977.

[13] Sh. Hu, N.S. Papageorgiou, "Handbook of multivalued analysis. Volume 1: theory", Kluwer Academic Publishers, Dordrecht, 1997.

14] N.I. Karachalios, H.E. Nistazakis, A.N. Yannacopoulos, Asymptotic behavior of solutions of complex discrete evolution equations: the discrete Ginzburg-Landau equation, Discrete Contin. Dyn. Syst., 19 (2007), 711-736 .

[15] N.I. Karachalios, N.I., A.N. Yannacopoulos, Global existence and compact attractors for the discrete nonlinear Schrödinger equations, J. Differential Equations, 217 (2005), 88-123.

[16] S. Kato, On existence and uniqueness conditions for nonlinear ordinary differential equations in Banach spaces, Funkcialaj Ekvacioj., 19 (1976), 239-245.

[17] F. Morillas, J. Valero, A Peano's theorem and attractors for lattice dynamical systems, Internat. J. Bifur. Chaos, 19 (2009).

[18] J.C. Oliveira, J.M. Pereira, G. Perla Menzala, Attractors for second order periodic lattices with nonlinear damping, J. Difference Equ. Appl., 14 (2008), no. 9, 899-921.

[19] B. Schmalfuß, Attractors for the non-autonomous dynamical systems, In "International Conference on Differential Equations, Vol. 1, 2 (Berlin, 1999)", World Sci. Publishing, River Edge, New-York, 2000, pp.684-689.

[20] B. Wang, Asymptotic behavior of non-autonomous lattice systems, J. Math. Anal. Appl. 331 (2007), $121-136$.

[21] Y. Wang, Y. Liu, Z. Wang, Random attractors for partly dissipative stochastic lattice dynamical systems, J. Difference Equ. Appl., 14 (2008), no. 8, 799-817

[22] C. Zhao, Sh. Zhou, Attractor of retarded first order lattice systems, Nonlinearity, 20 (2007), 19872006.

[23] S. Zhou, Attractors and approximations for lattice dynamical systems, J. Differential Equations 200 (2004), 342-368.

[24] S. Zhou, W. Shi, Attractors and dimension of dissipative lattice systems, J. Differential Equations 224 (2006), 172-204. 